\title{
Insights into the Phytochemical and Multifunctional Biological Profile of Spices from the Genus Piper
}

\author{
Simon Vlad Luca ${ }^{1}$, Katarzyna Gaweł-Bęben ${ }^{2, *} *$, Marcelina Strzępek-Gomółka ${ }^{2}{ }^{\circledR}$, Karolina Czech ${ }^{2}$,

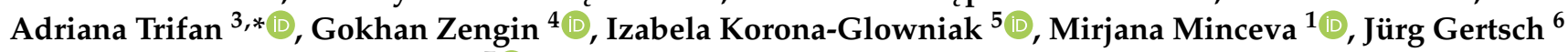 \\ and Krystyna Skalicka-Woźniak ${ }^{7}$ (1)
}

check for

updates

Citation: Luca, S.V.; Gaweł-Bęben, K.; Strzępek-Gomółka, M.; Czech, K.; Trifan, A.; Zengin, G.; KoronaGlowniak, I.; Minceva, M.; Gertsch, J.; Skalicka-Woźniak, K. Insights into the Phytochemical and Multifunctional Biological Profile of Spices from the Genus Piper. Antioxidants 2021, 10, 1642. https://doi.org/10.3390/ antiox10101642

Academic Editor: Evangelos Zoidis

Received: 5 October 2021

Accepted: 17 October 2021

Published: 19 October 2021

Publisher's Note: MDPI stays neutral with regard to jurisdictional claims in published maps and institutional affiliations.

Copyright: (c) 2021 by the authors. Licensee MDPI, Basel, Switzerland. This article is an open access article distributed under the terms and conditions of the Creative Commons Attribution (CC BY) license (https:// creativecommons.org/licenses/by/ $4.0 /)$.
1 Biothermodynamics, TUM School of Life Sciences, Technical University of Munich, 85354 Freising, Germany; vlad.luca@tum.de (S.V.L.); mirjana.minceva@tum.de (M.M.)

2 Department of Cosmetology, University of Information Technology and Management in Rzeszów, 35-225 Rzeszów, Poland; mstrzepek@wsiz.edu.pl (M.S.-G.); kczech@wsiz.edu.pl (K.C.)

3 Department of Pharmacognosy, Grigore T. Popa University of Medicine and Pharmacy Iasi, 700115 Iasi, Romania

4 Physiology and Biochemistry Research Laboratory, Department of Biology, Science Faculty, Selcuk University, Konya 42130, Turkey; gokhanzengin@selcuk.edu.tr

5 Department of Pharmaceutical Microbiology, Faculty of Pharmacy, Medical University of Lublin, Chodzki Street 1, 20-093 Lublin, Poland; iza.glowniak@umlub.pl

6 Institute of Biochemistry and Molecular Medicine, University of Bern, CH-3012 Bern, Switzerland; juerg.gertsch@ibmm.unibe.ch

7 Independent Laboratory of Natural Products Chemistry, Medical University of Lublin, 20-093 Lublin, Poland; kskalicka@pharmacognosy.org

* Correspondence: kagawel@wsiz.edu.pl (K.G.-B.); adriana.trifan@umfiasi.ro (A.T.)

Abstract: Piper spices represent an inexhaustible reservoir of bioactive compounds that may act as drug leads in natural product research. The aim of this study was to investigate a series of methanolic fruit extracts obtained from P. nigrum (black, green, white and red), P. longum and $P$. retrofractum in comparative phytochemical and multi-directional biological (antimicrobial, antioxidant, anti-enzymatic and anti-melanogenic) assays. The metabolite profiling revealed the presence of 17 piperamides, with a total content of $247.75-591.42 \mathrm{mg}$ piperine equivalents/g. Among the 22 tested microorganism strains, Piper spices were significantly active (MIC $<0.1 \mathrm{mg} / \mathrm{mL}$ ) against the anaerobes Actinomyces israelii and Fusobacterium nucleatum. The antioxidant and anti-enzymatic activities were evidenced in DPPH (10.64-82.44 mg TE/g) and ABTS (14.20-77.60 mg TE/g) radical scavenging, CUPRAC (39.94-140.52 mg TE/g), FRAP (16.05-77.00 mg TE/g), chelating (0-34.80 mg EDTAE/g), anti-acetylcholinesterase (0-2.27 mg GALAE/g), anti-butyrylcholinesterase (0.60-3.11 mg GALAE/g), anti-amylase (0.62-1.11 mmol ACAE/g) and anti-glucosidase (0-1.22 mmol ACAE/g) assays. Several Piper extracts ( $10 \mu \mathrm{g} / \mathrm{mL}$ ) inhibited both melanin synthesis (to $32.05-60.65 \%$ of $\alpha \mathrm{MSH}+$ cells) and release (38.06-45.78\% of $\alpha \mathrm{MSH}+$ cells) in $\alpha \mathrm{MSH}$-stimulated B16F10 cells, partly explained by their tyrosinase inhibitory properties. Our study uncovers differences between Piper spices and sheds light on their potential use as nutraceuticals or cosmeceuticals for the management of different diseases linked to bacterial infections, Alzheimer's dementia, type 2 diabetes mellitus or hyperpigmentation.

Keywords: Piper; piperamides; antimicrobial; anti-tyrosinase; anti-melanogenic; natural bioactive agents

\section{Introduction}

The genus Piper (Piperaceae, pepper) gathers around 1500-2000 species of herbs, shrubs, vines or trees, widely distributed in the tropical regions of southeast Asia and Oceania, Western and Central Africa, Central and South America [1,2]. Numerous members of the genus are valued as spices and have been used for thousands of years as flavor, color and aroma enhancers and preservatives [3]. Considered the 'king of spices' due to its massive share in the global spice market, $P$. nigrum L. is also used in traditional 
Chinese and Ayurvedic medicine to treat fever, colds, colic and gastrointestinal ailments [4]. Nevertheless, the fruits of $P$. nigrum (peppercorns) are differently processed, leading to numerous commercial varieties. For instance, black peppers represent the unripe berries allowed to dry until a wrinkled dark layer is formed, whereas white peppers are ripe fruits from which the outer layer has been removed. Green peppers are unripe berries picked at the same stage of ripeness as black peppercorns, while red peppers are unhulled ripe berries; both green and red peppercorns are dried under special color-preserving conditions [3].

The oblong, blunt and blackish-green fruits of P. longum L. are also among the most important species in the genus, being ethnomedicinally recommended as analgesic, immunemodulator, aphrodisiac or emmenagogue to treat respiratory or gastrointestinal disorders, post-partum hemorrhage, epilepsy, diabetes and rheumatoid arthritis [5,6]. Also elongated in shape, the fruits of P. retrofractum Vahl. (syn. P. chaba Hunter, P. officinarum (Miq.) C. DC.) are traditionally used as digestive, tonic, carminative in asthma, bronchitis, gastrointestinal ulcers, diarrhea or postpartum hemorrhage [1,7].

Spices from the genus Piper represent a promising lead in natural product research, as extracts or extract-derived bioactive compounds have been shown to act as multiple pharmacological agents with antioxidant, antimicrobial, anti-proliferative, anti-inflammatory, anti-hyperglycemic, anti-hyperlipidemic, cardio-vasculo-protective and neuroprotective effects $[4,6]$. Among the diverse specialized metabolites produced by the genus Piper (e.g., essential oils, flavonoids, lignans), piperamides are considered the key bioactive molecules [2]. As the main constituent of P. nigrum and P. longum, piperine was shown to possess analgesic, anti-depressant, anti-inflammatory and hepatoprotective effects in cell-free, cell-based and animal models [8]. Acyl coenzyme A-cholesterol acyltransferase, 5' adenosine monophosphate-activated protein kinase-p38 mitogen-activated protein kinase signaling pathway, P-glycoprotein and different ion channels are among the most promising molecular targets of piperine $[9,10]$. In addition, other piperamides, such as pipernonaline, retrofractamide B, guineensine, $\mathrm{N}$-isobutyl-2,4-octadecadienamide, $\mathrm{N}$-isobutyl-2,4,14eicosatrienamide and piperanine exhibited gastro-protective effects in indomethacin- and ethanol-induced gastric lesions in rats, whereas piperolein $B$, retrofractamide $C$, pipernonaline and dehydropipernonaline were reported to act as diacylglycerol acyltransferase inhibitors, with putative implications in the treatment of obesity [11]. Furthermore, guineensine has been shown to be a nanomolar endocannabinoid reuptake inhibitor with potent in vivo cannabimimetic effects $[12,13]$.

As a continuation of our multi-group research efforts to screen for new phytochemical drug leads for the management of infectious diseases, Alzheimer's disease, type 2 diabetes mellitus and skin disorders, the biological and metabolite profiling of P. nigrum (black, green, white and red), as well as of P. longum and P. retrofractum, was performed. The comprehensive phytochemical characterization of Piper spices was carried out by stateof-the-art spectro-chromatographic techniques, whereas the antibacterial and antifungal properties were assessed on a panel of reference microorganisms. The antioxidant and anti-enzymatic activities were investigated with regard to radical scavenging, metal reducing and chelating, anti-cholinesterase, anti-amylase, anti-glucosidase and anti-tyrosinase assays. Lastly, the anti-melanogenic effects were evaluated in murine melanoma B16F10 cells stimulated with $\alpha$-melanocyte stimulating hormone ( $\alpha \mathrm{MSH})$.

\section{Materials and Methods}

\subsection{Plant Material and Extraction}

Dried fruits (peppercorns) of various Piper spices (Table 1) were bought from various local European markets, identified by the authors (A.T., K.S.W.) and stored in Department of Pharmacognosy, Grigore T. Popa University of Medicine and Pharmacy Iasi (Romania). The dried peppercorns ( $20 \mathrm{~g}$ from each material) were pulverized with a grinding machine (Rommelsbacher ElektroHausgeräte $\mathrm{GmbH}$, Dinkelsbühl, Germany) and subjected to ultrasound-assisted extraction with methanol $(200 \mathrm{~mL})$ for $30 \mathrm{~min}$ at $40{ }^{\circ} \mathrm{C}$, for three 
consecutive times. The filtered and pooled extracts were evaporated to dryness. The extraction yields are provided for each sample in Table 1.

Table 1. Extraction yields, total phenolic and flavonoid contents in the methanolic fruit extracts obtained from different spices of genus Piper.

\begin{tabular}{|c|c|c|c|c|c|}
\hline \multicolumn{2}{|c|}{ Sample } & \multirow{2}{*}{$\begin{array}{l}\text { Voucher } \\
\text { PN1/102019 }\end{array}$} & \multirow{2}{*}{$\begin{array}{c}\begin{array}{c}\text { Extraction } \\
\text { Yield }\end{array} \\
10.5 \%\end{array}$} & \multirow{2}{*}{$\begin{array}{c}\text { TPC (mg GAE/g) } \\
40.96 \pm 0.42^{b}\end{array}$} & \multirow{2}{*}{$\begin{array}{r}\text { TFC (mg RE/g) } \\
10.03 \pm 0.22^{b}\end{array}$} \\
\hline \multirow{6}{*}{ P. nigrum $\mathrm{L}$. } & Black1 & & & & \\
\hline & Black2 & PN5/102019 & $10.9 \%$ & $40.80 \pm 0.45^{b}$ & $9.12 \pm 0.32^{b}$ \\
\hline & Black3 & PN6/102019 & $11.9 \%$ & $36.71 \pm 0.18^{c}$ & $1.44 \pm 0.15^{\mathrm{e}}$ \\
\hline & White & PN2/1020219 & $7.7 \%$ & $42.11 \pm 1.06^{b}$ & $4.66 \pm 0.30^{c}$ \\
\hline & Green & PN3/102019 & $12.5 \%$ & $58.90 \pm 1.84^{a}$ & $18.37 \pm 0.27^{\mathrm{a}}$ \\
\hline & Red & PN4/102019 & $14.5 \%$ & $41.41 \pm 1.00^{b}$ & $5.42 \pm 0.32^{c}$ \\
\hline \multicolumn{2}{|c|}{ P. longum L. } & PL1/112019 & $18.3 \%$ & $29.53 \pm 0.25^{d}$ & $3.01 \pm 0.03^{\mathrm{d}}$ \\
\hline \multicolumn{2}{|c|}{ P. retrofractum Vahl. } & PR1/112019 & $10.1 \%$ & $32.60 \pm 0.22 e^{e}$ & $19.70 \pm 0.13^{d}$ \\
\hline
\end{tabular}

Data are presented as mg gallic acid equivalents (GAE)/g extract for the total phenolic content (TPC) and mg rutin equivalents (RE)/g extract for total flavonoid content (TFC) and represent the mean values \pm S.D. of three determinations; values in the same column sharing different superscript letters are significantly different at $p<0.05$.

\subsection{Phytochemical Characterization}

Total phenolic content (TPC) and total flavonoid content (TFC) of Piper methanolic extracts were determined as previously described [14,15], with the results expressed as $\mathrm{mg}$ gallic acid equivalents (GAE)/g extract for TPC and $\mathrm{mg}$ rutin equivalents (RE)/g extract for TFC. The profiling of piperamides was performed by liquid chromatography with high resolution tandem mass spectrometry (LC-HRMS/MS), based on a method detailed by Luca et al. [2]. The quantitative estimation of piperamides was carried out by liquid chromatography with diode array detection (LC-DAD), as previously presented [2]; the data are reported as $\mathrm{mg}$ piperine equivalents $(\mathrm{PE}) / \mathrm{g}$ extract.

\subsection{Antimicrobial Assay}

The methanolic extracts of Piper spices were screened for their antibacterial and antifungal activities by micro-dilution method according to the European Committee on Antimicrobial Susceptibility Testing [16]. Mueller-Hinton (MH) broth and MH broth with $5 \%$ lysed sheep blood were used for the growth of non-fastidious and fastidious bacteria, respectively, whereas MH broth with $2 \%$ glucose was used for the growth of fungi. Minimum inhibitory concentrations (MIC) of the samples were determined in a panel of reference microorganisms. The following strains were used: Gram-positive bacteria (Staphylococcus aureus ATCC 25923, Staphylococcus epidermidis ATCC 12228, Micrococcus luteus ATCC 10240, Bacillus cereus ATCC 10876, Enterococcus faecalis ATCC 29212, Streptococcus mutans ATCC 25175, Actinomyces israelii ATCC 10049 and Clostridium perfringens ATCC 13124); Gramnegative bacteria (Campylobacter jejunii ATCC 33291, Salmonella Typhimurium ATCC14028, Escherichia coli ATCC 25922, Proteus mirabilis ATCC 12453, Klebisiella pneumoniae ATCC 13883, Pseudomonas aeruginosa ATCC 90271, Bacteroides fragilis ATCC 10240, Prevotella intermedia ATCC 25611, Fusobacterium nucleatum ATCC 25586 and Veillonella parvula ATCC 10790); fungi (Candida glabrata ATCC 90030, C. albicans ATCC 102231 and C. parapsilosis ATCC 22019).

\subsection{Antioxidant, Cholinesterase, Amylase and Glucosidase Assays}

The antioxidant activity of Piper samples was determined by 1,1-diphenyl-2-picryhydrazyl (DPPH) and 2,2'-azino-bis(3-ethylbenzothiazoline-6-sulfonic acid) (ABTS) radical scavenging, cupric reducing antioxidant capacity (CUPRAC), ferric reducing antioxidant power (FRAP), metal chelating (MCA) and phosphomolybdenum (PBD) assays [14,15]. DPPH, ABTS, CUPRAC and FRAP activities are expressed as mg trolox equivalents (TE)/g extract. 
In MCA, the data were presented as mg EDTA equivalents (EDTAE)/g extract, whereas in PBD assay, the results are provided are mmol TE/g extract. Acetylcholinesterase (AChE) and butyrylcholinesterase (BChE) inhibition was assessed as described in [14,15] and expressed as mg galanthamine equivalents (GALAE)/g extract, while amylase and glucosidase inhibition $[14,15]$ is reported as $\mathrm{mg}$ acarbose equivalents (ACAE) $/ \mathrm{g}$ extract.

\subsection{Cell Viability Assay}

Murine melanoma B16F10 cells (ATCC ${ }^{\circledR}$ CRL-6475, LGC Standards, Łomianki, Poland) and immortalized human keratinocyte HaCaT cells (Cell Lines Service GmbH, Eppelheim, Germany) were maintained in Dulbecco's Modified Eagle's Medium (DMEM) containing $4.5 \mathrm{~g} / \mathrm{L}$ glucose and supplemented with $10 \%$ fetal bovine serum (FBS). The cells were cultured at $37{ }^{\circ} \mathrm{C}$ in a humidified atmosphere with $5 \% \mathrm{CO}_{2}$. Neutral red uptake (NRU) assay was performed as described previously by Repetto et al. [17]. Briefly, after seeding in 96-well plates $\left(3 \times 10^{3}\right.$ cells / well), cells were grown overnight and treated with different concentrations of Piper methanolic extracts $(10-200 \mu \mathrm{g} / \mathrm{mL})$ or piperine $(10-200 \mu \mathrm{g} / \mathrm{mL})$. Control cells, containing appropriate volumes of the solvent (DMSO), were kept under the same conditions. After $72 \mathrm{~h}$ (for B16F10 cells) or $48 \mathrm{~h}$ (for HaCaT cells), the cells were incubated for $3 \mathrm{~h}$ with neutral red solution $(33 \mu \mathrm{g} / \mathrm{mL})$ in conditioned medium containing $1 \%$ FBS. The cell morphology was examined with an inverted microscope (Nikon Eclipse, Nikon, Japan) and documented using an Invenio II camera (DEltaPix, Smørum, Denmark). The cells were then rinsed with Dulbecco's phosphate buffered saline (DPBS) and lysed using acidified ethanol solution (50\% $v / v$ ethanol, $1 \% v / v$ acetic acid and $49 \% \mathrm{H}_{2} \mathrm{O}$ ). The absorbance of the released neutral red was measured at $\lambda=540 \mathrm{~nm}$ using a FilterMax F5 microplate reader (Molecular Devices, San Jose, CA, USA) and corrected by the absorbance at $\lambda=620 \mathrm{~nm}$. Mean measured values for the lysate from control cells were set as $100 \%$ and used to calculate the percentage of cell viability in the other samples.

\subsection{Melanin Assay}

After seeding in 6-well plates $\left(0.5 \times 10^{5}\right.$ cells / well), B16F10 cells were grown over night and treated with Piper methanolic extracts $(10 \mu \mathrm{g} / \mathrm{mL})$, piperine $(10 \mu \mathrm{g} / \mathrm{mL})$ or the positive control kojic acid $(10 \mu \mathrm{g} / \mathrm{mL})$. Melanin production was stimulated with $\alpha \mathrm{MSH}$ $(10 \mathrm{nM})$. After $72 \mathrm{~h}$, the conditioned medium and cell pellets were collected. Negative control cells, containing appropriate volumes of solvent (DMSO), were kept under the same conditions, but in the absence of $\alpha \mathrm{MSH}$ stimulation. Next, cell pellets were dissolved in $1 \mathrm{~N} \mathrm{NaOH}$ and incubated for $2 \mathrm{~h}$ at $80^{\circ} \mathrm{C}$. Conditioned media and cell lysates were then transferred to a 96-well plate and the absorbance was measured at $\lambda=405 \mathrm{~nm}$ using the FilterMax F5 microplate reader. The content of protein in cell lysates was established by Bradford assay [18]. The melanin released in the conditioned medium and the melanin content in cell lysates ( $\mu$ g melanin/mg protein) were calculated with the help of synthetic melanin calibration curves. Mean measured values obtained for $\alpha \mathrm{MSH}$-stimulated control cells without the tested samples $(\alpha \mathrm{MSH}+)$ were set as $100 \%$ and used to calculate the percentage of melanin release/content in the other samples.

\subsection{Tyrosinase Assay}

\subsubsection{Mushroom Tyrosinase Assay}

The inhibition of the monophenolase and diphenolase activities of mushroom tyrosinase was performed as previously described $[19,20]$. For the monophenolase inhibitory assay, $100 \mathrm{mM}$ phosphate buffer (PBS, $\mathrm{pH}=6.8,80 \mu \mathrm{L}$ ) was mixed with $20 \mu \mathrm{L}$ of Piper methanolic extracts $(10 \mu \mathrm{g} / \mathrm{mL})$, piperine $(10 \mu \mathrm{g} / \mathrm{mL})$ or kojic acid $(10 \mu \mathrm{g} / \mathrm{mL})$. After incubation at $25{ }^{\circ} \mathrm{C}$ for $10 \mathrm{~min}$ with $500 \mathrm{U} / \mathrm{mL}$ mushroom tyrosinase $(20 \mu \mathrm{L}), 2 \mathrm{mM}$ L-tyrosine $(80 \mu \mathrm{L})$ was added to the samples and incubated at $25^{\circ} \mathrm{C}$ for additional $20 \mathrm{~min}$. In the case of diphenolase inhibitory assay, PBS $(120 \mu \mathrm{L})$ and samples $(20 \mu \mathrm{L})$ were incubated for $10 \mathrm{~min}$ at $25^{\circ} \mathrm{C}$ with mushroom tyrosinase $(80 \mu \mathrm{L})$. Following the addition of $4 \mathrm{mM}$ L-DOPA $(40 \mu \mathrm{L})$, the samples were further incubated for additional $20 \mathrm{~min}$ at $25^{\circ} \mathrm{C}$. In both 
assays, the control, containing appropriate volumes of solvent (DMSO), was obtained under the same conditions. Next, the formation of dopachrome was measured spectrophotometrically at $\lambda=450 \mathrm{~nm}$ using the FilterMax F5 microplate reader. Mean measured values obtained for the solvent control were set as $100 \%$ and used to calculate the percentage of enzyme activity in the other samples.

\subsubsection{Murine Tyrosinase Assay}

The inhibition of murine tyrosinase activity was assessed using B16F10 cell lysate, as previously described $[19,20]$. The B16F10 cells $\left(8 \times 10^{6}\right)$ were lysed for $1 \mathrm{~h}$ with $1 \%$ Triton $\mathrm{X}-100$ in PBS $(5 \mathrm{~mL})$ in an ultrasound ice-cold water bath. After centrifugation (10 min at $13,000 \mathrm{rpm})$, the supernatant was collected as murine tyrosine solution and the protein concentration was measured using a DC Protein Assay kit (Bio-Rad, Hercules, CA, USA). Cell lysate containing $20 \mu \mathrm{g}$ protein was mixed with $20 \mu \mathrm{L}$ of Piper methanolic extracts $(10 \mu \mathrm{g} / \mathrm{mL})$, piperine $(10 \mu \mathrm{g} / \mathrm{mL})$ or kojic acid $(10 \mu \mathrm{g} / \mathrm{mL}), 4 \mathrm{mM} \mathrm{L}-\mathrm{DOPA}(40 \mu \mathrm{L})$ and PBS (up to $200 \mu \mathrm{L}$ ). The control, containing appropriate volumes of solvent (DMSO), was obtained under the same conditions. After incubation for $4 \mathrm{~h}$ at $37^{\circ} \mathrm{C}$, the absorbance was measured spectrophotometrically at $\lambda=450 \mathrm{~nm}$ using the FilterMax F5. Mean measured values obtained for the solvent control were set as $100 \%$ and used to calculate the percentage of enzyme activity in the other samples.

\subsection{Statistical Analysis}

All non-cell-based tests were performed in three replicates, and the results are expressed as mean \pm standard deviation (S.D.). The cell-based assays were performed in triplicate, with data representative for at least three individual experiments and expressed as mean \pm standard error of mean (S.E.M.). Statistical analysis was performed in OriginPro2020 (OriginLab) using ANOVA with Turkey's post hoc test; $p<0.05$ was considered statistically significant.

\section{Results and Discussion}

\subsection{Phytochemical Characterization}

Piper fruits are acknowledged as valuable and rich sources of a wide range of specialized metabolites, such as piperamides, flavonoids, lignans and essential oils [6]. In the current work, the methanolic fruits extracts of P. nigrum, P. longum and P. retrofractum were phytochemically characterized with regard to TPC, TFC as well as the piperamides' profile (LC-HRMS/MS) and content (LC-DAD).

TPC was generally higher in the six varieties of black pepper (36.71-58.90 mg GAE/g) than in P. longum (29.53 $\mathrm{mg}$ GAE/g) and P. retrofractum (32.60 $\mathrm{mg}$ GAE/g) (Table 1). TFC showed a high variability, with $P$. retrofractum $(19.70 \mathrm{mg} \mathrm{RE} / \mathrm{g})$ displaying the highest values, followed by green pepper (18.37 $\mathrm{mg} \mathrm{RE} / \mathrm{g})$; on the contrary, black pepper 3 (1.44 mg RE/g) exhibited a very low TFC (Table 1). Our results are comparable with previous literature data. For instance, Zarai et al. [21] reported $37.48 \mathrm{mg}$ GAE/g and $3.01 \mathrm{mg} / \mathrm{g}$ quercetin equivalents $/ \mathrm{g}$ in the methanol extract of $P$. nigrum.

The LC-HRMS/MS profiling of piperamides was performed according to dereplication strategies carefully presented in a previous work for a series of hexane Piper extracts [2]. To estimate the piperamides' content, the chromatographic and spectrometric LC-HRMS/MS identification data (UV, HRMS, HRMS/MS, molecular formula) from Table 2 were correlated with the LC-DAD data, as detailed in [2]. 
Table 2. LC-HRMS/MS data of piperamides tentatively identified the methanolic fruit extracts obtained from different spices of genus Piper.

\begin{tabular}{|c|c|c|c|c|c|}
\hline No. & Compound & $\begin{array}{c}\text { UV } \\
{[\mathrm{nm}]}\end{array}$ & $\begin{array}{c}\text { HRMS (+) } \\
\quad[m / z]\end{array}$ & MF & $\begin{array}{c}\text { HRMS/MS (+) } \\
{[m / z]}\end{array}$ \\
\hline 1 & Piperolactam C & $220 ; 340$ & 310.1075 & $\mathrm{C}_{18} \mathrm{H}_{15} \mathrm{NO}_{4}$ & $193.0750 ; 120.0395$ \\
\hline 2 & Piperlongumine & $230 ; 330$ & 318.1360 & $\mathrm{C}_{17} \mathrm{H}_{19} \mathrm{NO}_{5}$ & $269.0957 ; 221.0805 ; 201.0527$ \\
\hline 3 & Piperyline & $255 ; 310 ; 342$ & 272.1291 & $\mathrm{C}_{16} \mathrm{H}_{17} \mathrm{NO}_{3}$ & $\begin{array}{c}201.0555 ; 173.0572 ; 143.0503 ; 135.0405 ; \\
115.0571\end{array}$ \\
\hline 4 & Piperlonguminine & $230 ; 340$ & 274.1452 & $\mathrm{C}_{16} \mathrm{H}_{19} \mathrm{NO}_{3}$ & $161.0576 ; 135.0452$ \\
\hline 5 & Piperine & $240 ; 310 ; 341$ & 286.1443 & $\mathrm{C}_{17} \mathrm{H}_{19} \mathrm{NO}_{3}$ & $201.0542 ; 173.0525 ; 135.0455 ; 112.0733$ \\
\hline 6 & Piperettines & $310 ; 345$ & 312.1605 & $\mathrm{C}_{19} \mathrm{H}_{21} \mathrm{NO}_{3}$ & $\begin{array}{c}227.0679 ; 199.0776 ; 169.0555 ; 141.085 ; \\
131.0405\end{array}$ \\
\hline 7 & Piperolein A & $260 ; 340$ & 316.1904 & $\mathrm{C}_{19} \mathrm{H}_{25} \mathrm{NO}_{3}$ & 231.1056; 161.0538; 135.0512 \\
\hline 8 & Pellitorine & $210 ; 260$ & 224.2015 & $\mathrm{C}_{14} \mathrm{H}_{25} \mathrm{NO}$ & $168.1414 ; 151.1170 ; 123.1196 ; 109.0603$ \\
\hline 9 & Pipercallosine & $210 ; 265 ; 310$ & 330.2049 & $\mathrm{C}_{20} \mathrm{H}_{27} \mathrm{NO}_{3}$ & $259.1312 ; 241.1301 ; 208.1725 ; 135.0490$ \\
\hline 10 & Dehydropipernonaline & $210 ; 265$ & 340.1919 & $\mathrm{C}_{21} \mathrm{H}_{25} \mathrm{NO}_{3}$ & $227.1001 ; 161.0554 ; 131.0414$ \\
\hline 11 & Pipernonaline & $215 ; 260 ; 305$ & 342.2065 & $\mathrm{C}_{21} \mathrm{H}_{27} \mathrm{NO}_{3}$ & $229.1564 ; 199.0916 ; 161.0593 ; 135.0471$ \\
\hline 12 & Neopeollitorine B & $210 ; 265$ & 236.2001 & $\mathrm{C}_{15} \mathrm{H}_{25} \mathrm{NO}$ & $151.1140 ; 109.0625$ \\
\hline 13 & Retrofractamide B & $210 ; 260$ & 356.2203 & $\mathrm{C}_{22} \mathrm{H}_{29} \mathrm{NO}_{3}$ & $\begin{array}{c}283.1309 ; 255.1371 ; 234.1822 ; 215.1868 ; \\
135.0433\end{array}$ \\
\hline 14 & Piperolein B & $210 ; 265 ; 305$ & 344.2205 & $\mathrm{C}_{21} \mathrm{H}_{29} \mathrm{NO}_{3}$ & $259.1299 ; 222.1829 ; 135.0395$ \\
\hline 15 & Piperundecalidine & $215 ; 270$ & 368.2223 & $\mathrm{C}_{23} \mathrm{H}_{29} \mathrm{NO}_{3}$ & $246.1807 ; 215.1046 ; 173.0559 ; 135.0411$ \\
\hline 16 & Guineensine & $215 ; 260 ; 315$ & 384.2586 & $\mathrm{C}_{24} \mathrm{H}_{33} \mathrm{NO}_{3}$ & $311.1985 ; 283.2033 ; 161.0754 ; 135.0577$ \\
\hline 17 & $\begin{array}{l}N \text {-Isobutyl-2,4,12- } \\
\text { octadecatrienamide }\end{array}$ & $220 ; 260$ & 334.3099 & $\mathrm{C}_{22} \mathrm{H}_{39} \mathrm{NO}$ & $261.2253 ; 233.2201$ \\
\hline
\end{tabular}

HRMS, high-resolution mass spectra; $M F$, molecular formula; $T_{R}$ retention time; $U V$, ultraviolet.

Piperolactam C (1) and piperlongumine (2) were solely found in P. retrofractum, whereas piperyline (3) was present only in P. nigrum $(5.81-15.40 \mathrm{mg} / \mathrm{g}$ ). The amount of piperlonguminine (4) varied from $2.72 \mathrm{mg} / \mathrm{g}$ in white pepper to $16.87 \mathrm{mg} / \mathrm{g}$ in P. longum. Piperine (5) was by far the most abundant constituent in almost analyzed samples, with its highest values in white and green peppers $(308.36-315.19 \mathrm{mg} / \mathrm{g})$; in comparison, P. retrofractum had $\sim 3$-fold lower values of piperine. Nevertheless, the major piperamide in P. retrofractum was represented by pellitorine $(8 ; 206.95 \mathrm{mg} / \mathrm{g})$, a constituent found in levels 5 to 25 times lower in the other spices. Quantified as sum of stereoisomers, piperettines (6) showed significantly high amounts in P. nigrum varieties $(21.51-70.60 \mathrm{mg} / \mathrm{g})$, while their content was very low in the other two investigated Piper species (Table 2).

Piperolein A (7) and piperolein B (14) were absent in P. longum, whereas their levels in the other spices varied from $5.84 \mathrm{mg} / \mathrm{g}$ (red pepper) to $28.97 \mathrm{mg} / \mathrm{g}$ (white pepper) and from $2.70 \mathrm{mg} / \mathrm{g}$ (P. retrofractum) to $17.26 \mathrm{mg} / \mathrm{g}$ (black pepper 1), respectively. Pipercallosine (9), pipernonaline (11; $47.62 \mathrm{mg} / \mathrm{g}$ ) and $\mathrm{N}$-Isobutyl-2,4,12-octadecatrienamide (17; $82.65 \mathrm{mg} / \mathrm{g}$ ) reached their highest values in P. longum. On the contrary, samples of P. nigrum were characterized by the highest amounts of dehydropipernonaline $(\mathbf{1 0} ; 22.23 \mathrm{mg} / \mathrm{g}$ ), neopeollitorine B (12; $7.89 \mathrm{mg} / \mathrm{g})$, retrofractamide B (13;22.07 mg/g), piperundecalidine $(15 ; 7.66 \mathrm{mg} / \mathrm{g})$ and guineensine $(\mathbf{1 6} ; 42.53 \mathrm{mg} / \mathrm{g})$. The total piperamide content revealed a variable profile from 247.75 to $591.42 \mathrm{mg} / \mathrm{g}$, in the following increasing order: red pepper $<$ P. retrofractum $<$ black pepper $3<$ P. longum $<$ white pepper $\sim$ black pepper $2<$ green pepper black pepper 1 (Table 3 ). 
Table 3. Piperamide content in the methanolic fruit extracts obtained from different spices of genus Piper.

\begin{tabular}{|c|c|c|c|c|c|c|c|c|c|}
\hline \multirow{3}{*}{ No. } & \multirow{3}{*}{ Compound } & \multicolumn{6}{|c|}{ P. nigrum } & \multirow{2}{*}{ P. longum } & \multirow{2}{*}{ P. retrofractum } \\
\hline & & Black 1 & Black 2 & Black 3 & White & Green & Red & & \\
\hline & & \multicolumn{8}{|c|}{ mg PE/g Extract } \\
\hline 2 & Piperlongumine & $\mathrm{nq}$ & nq & $\mathrm{nq}$ & $\mathrm{nq}$ & nq & $\mathrm{nq}$ & $\mathrm{nq}$ & $14.19 \pm 0.14^{\mathrm{a}}$ \\
\hline 3 & Piperyline & $11.66 \pm 0.17^{c}$ & $12.68 \pm 0.16^{b}$ & $8.62 \pm 0.18^{\mathrm{e}}$ & $10.90 \pm 0.08^{d}$ & $15.40 \pm 0.34^{\mathrm{a}}$ & $5.81 \pm 0.07^{\mathrm{f}}$ & $\mathrm{nq}$ & nq \\
\hline 4 & Piperlonguminine & $4.90 \pm 0.12^{\mathrm{d}}$ & $4.84 \pm 0.26^{\mathrm{d}}$ & $2.83 \pm 0.13^{\mathrm{e}}$ & $2.72 \pm 0.15^{\mathrm{f}}$ & $6.01 \pm 0.14^{c}$ & $5.10 \pm 0.13^{\mathrm{d}}$ & $16.87 \pm 0.13^{\mathrm{a}}$ & $12.93 \pm 0.03^{b}$ \\
\hline 5 & Piperine & $276.70 \pm 0.82^{d}$ & $299.63 \pm 0.99^{c}$ & $293.07 \pm 0.71^{\mathrm{c}}$ & $308.36 \pm 0.50^{b}$ & $315.19 \pm 1.12^{\mathrm{a}}$ & $154.03 \pm 0.21^{\mathrm{f}}$ & $249.79 \pm 0.99^{\mathrm{e}}$ & $93.86 \pm 0.13 \mathrm{~g}$ \\
\hline 7 & Piperolein A & $28.83 \pm 1.60^{\mathrm{a}}$ & $17.96 \pm 0.28^{c}$ & $9.93 \pm 0.16^{\mathrm{d}}$ & $28.97 \pm 0.66^{\mathrm{a}}$ & $23.90 \pm 0.54^{b}$ & $5.84 \pm 0.12^{\mathrm{e}}$ & $\mathrm{nq}$ & $23.00 \pm 1.21^{b}$ \\
\hline 8 & Pellitorine & $39.68 \pm 0.14^{b}$ & $15.95 \pm 0.10^{\mathrm{e}}$ & $22.41 \pm 0.26^{\mathrm{d}}$ & $26.11 \pm 0.17^{c}$ & $18.78 \pm 0.15^{\mathrm{e}}$ & $9.33 \pm 0.07^{\mathrm{f}}$ & $7.90 \pm 0.35 \mathrm{~g}$ & $206.95 \pm 0.22^{a}$ \\
\hline 9 & Pipercallosine & $3.72 \pm 0.51^{\mathrm{c}}$ & $3.28 \pm 0.15^{\mathrm{d}}$ & $3.02 \pm 0.06^{\mathrm{d}}$ & $4.72 \pm 0.37^{\mathrm{b}}$ & $4.57 \pm 0.18^{b}$ & $2.04 \pm 0.04^{\mathrm{e}}$ & $9.25 \pm 0.47^{\mathrm{a}}$ & $\mathrm{nq}$ \\
\hline 10 & Dehydropipernonaline & $22.23 \pm 0.29^{a}$ & $11.18 \pm 0.09^{\mathrm{e}}$ & $18.50 \pm 0.46^{\mathrm{b}}$ & $17.12 \pm 0.17^{\mathrm{c}}$ & $12.77 \pm 0.12^{\mathrm{d}}$ & $2.68 \pm 0.04^{\mathrm{f}}$ & $12.71 \pm 0.29^{\mathrm{d}}$ & $\mathrm{nq}$ \\
\hline 11 & Pipernonaline & $3.37 \pm 0.12^{c}$ & $\mathrm{nq}$ & $2.14 \pm 0.20^{\mathrm{d}}$ & $5.60 \pm 0.44^{\mathrm{b}}$ & $\mathrm{nq}$ & $\mathrm{nq}$ & $47.62 \pm 0.29^{a}$ & $\mathrm{nq}$ \\
\hline 12 & Neopeollitorine B & $7.89 \pm 0.65^{\mathrm{a}}$ & $3.26 \pm 0.10^{c}$ & $2.73 \pm 0.20^{\mathrm{d}}$ & $2.31 \pm 0.36^{\mathrm{d}}$ & $3.14 \pm 0.01^{c}$ & $\mathrm{nq}$ & $\mathrm{nq}$ & $6.51 \pm 0.25^{b}$ \\
\hline 13 & Retrofractamide B & $22.07 \pm 0.57^{\mathrm{a}}$ & $13.28 \pm 0.21^{\mathrm{d}}$ & $6.14 \pm 0.06^{\mathrm{f}}$ & $6.75 \pm 0.31^{\mathrm{e}}$ & $19.75 \pm 0.21^{b}$ & $12.65 \pm 0.12^{\mathrm{d}}$ & $14.63 \pm 0.07^{c}$ & $\mathrm{nq}$ \\
\hline 14 & Piperolein B & $17.26 \pm 0.10^{\mathrm{a}}$ & $12.46 \pm 0.27^{\mathrm{d}}$ & $11.02 \pm 0.01^{\mathrm{e}}$ & $13.17 \pm 0.24^{\mathrm{c}}$ & $15.35 \pm 0.20^{\mathrm{b}}$ & $3.20 \pm 0.06^{f}$ & $\mathrm{nq}$ & $2.70 \pm 0.01^{g}$ \\
\hline \multirow[t]{2}{*}{17} & $\begin{array}{l}N \text {-Isobutyl-2,4,12- } \\
\text { octadecatrienamide }\end{array}$ & $32.31 \pm 0.37^{\mathrm{d}}$ & $14.18 \pm 0.21^{\mathrm{e}}$ & $49.44 \pm 0.33^{\mathrm{b}}$ & $13.61 \pm 0.19^{\mathrm{f}}$ & $37.62 \pm 0.20^{c}$ & $12.64 \pm 0.05^{\mathrm{g}}$ & $82.65 \pm 0.25^{\mathrm{a}}$ & $1.98 \pm 0.05^{\mathrm{h}}$ \\
\hline & Total piperamides & $591.42 \pm 4.01^{\mathrm{a}}$ & $525.35 \pm 4.10^{b}$ & $588.42 \pm 6.44^{\mathrm{a}}$ & $247.75 \pm 0.87^{\mathrm{f}}$ & $530.67 \pm 1.49^{b}$ & $453.58 \pm 7.63^{d}$ & $475.89 \pm 1.05^{c}$ & $423.72 \pm 2.49^{\mathrm{e}}$ \\
\hline
\end{tabular}

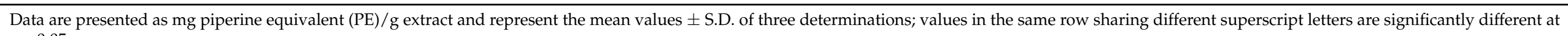
$p<0.05$. 


\subsection{Antimicrobial Activity}

The extensive usage of antibiotics and the rapid emergence of multi-drug resistant pathogens represent serious issues for modern medicine. Numerous solutions are under current investigation, including the screening and identification of novel plant-based antimicrobials with superior efficiency and safety profiles [22]. In the present study, the antimicrobial activity of the eight methanolic fruit extracts of Piper spp. was assessed by micro-dilution method, with results reported as MIC values (Table 4). The sensitivity of the tested strains varied based on the Piper variety/species, microorganism group or species. The criteria proposed by Kuete and Efferth [23] were used to categorize the observed antimicrobial activity into three groups: significant activity (MIC $<0.1 \mathrm{mg} / \mathrm{mL}$ ), moderate activity $(0.1<$ MIC $<0.625 \mathrm{mg} / \mathrm{mL})$ and weak $/$ no activity $(\mathrm{MIC}>0.625 \mathrm{mg} / \mathrm{mL})$. For instance, a moderate activity (MIC $=0.125-0.5 \mathrm{mg} / \mathrm{mL}$ ) was noticed against $S$. aureus for black peppers 2 and 3, white pepper, green pepper and P. retrofractum, whereas only green and red peppers were found to be active against S. epidermidis. Except for P. longum, all other investigated spices presented a moderate activity against $M$. luteus, with black peppers 1 and 2 and green pepper as the most potent agents. Black peppers 1 and 3, white pepper and P. retrofractum exhibited MIC values of $0.5 \mathrm{mg} / \mathrm{mL}$ against Bacillus cereus. The anaerobic $A$. israelii showed a particular sensitivity toward all Piper samples (MIC $=0.0625-0.5 \mathrm{mg} / \mathrm{mL}$ ), with black pepper 3 and white pepper displaying the strongest inhibitory effects. The investigated spices also demonstrated weak activity against aerobic Gram-negative bacteria (MIC $>2 \mathrm{mg} / \mathrm{mL}$ ), whereas the anaerobic Gram-negative bacteria (B. fragilis, P. intermedia, F. nucleatum, V. parvula) were considerably more sensitive. For instance, a MIC value of $0.0625 \mathrm{mg} / \mathrm{mL}$ was noticed for black pepper 3, green pepper and $P$. retrofractum against $F$. nucleatum, while black peppers 1-2, white and red peppers exhibited a MIC value of $0.125 \mathrm{mg} / \mathrm{mL}$ against the same pathogen. Concerning the yeast strains, all spices (except for P. longum) displayed moderate antifungal activity $(\mathrm{MIC}=0.25-0.5 \mathrm{mg} / \mathrm{mL}$ ), but only against $C$. parapsilosis (Table 4 ).

Table 4. Antimicrobial activity of the methanolic fruit extracts obtained from different spices of genus Piper.

\begin{tabular}{|c|c|c|c|c|c|c|c|c|c|}
\hline \multirow{3}{*}{ Microbial Species } & \multicolumn{6}{|c|}{ P. nigrum } & \multirow{2}{*}{ P. longum } & \multirow{2}{*}{ P. retrofractum } & \multirow{2}{*}{ Reference drug } \\
\hline & Black 1 & Black 2 & Black 3 & White & Green & Red & & & \\
\hline & \multicolumn{8}{|c|}{ MIC (mg/mL) } & MIC (mg/L) \\
\hline S. aureus ATCC 25923 & 4 & 0.25 & 0.5 & 0.125 & 0.5 & 1 & 4 & 0.25 & $0.98^{1}$ \\
\hline S. epidermidis ATCC 12228 & $>4$ & 2 & 4 & 4 & 0.125 & 0.5 & 4 & 4 & $0.98^{1}$ \\
\hline M. luteus ATCC 10240 & 0.125 & 0.125 & 0.25 & 0.125 & 0.25 & 0.5 & 1 & 0.5 & $0.12^{1}$ \\
\hline B. cereus ATCC 10876 & 0.5 & 1 & 0.5 & 0.5 & 2 & 2 & 4 & 0.5 & $0.98^{1}$ \\
\hline E. faecalis ATCC 29212 & $>4$ & $>4$ & $>4$ & 4 & 4 & $>4$ & 4 & $>4$ & $1.95^{1}$ \\
\hline S. mutans ATCC 25175 & 4 & 4 & 4 & 4 & 4 & 4 & $>4$ & 4 & $0.98^{1}$ \\
\hline A. israelii ATCC 10049 & 0.125 & 0.125 & 0.0625 & 0.0625 & 0.125 & 0.5 & 0.5 & 0.25 & $0.5^{1}$ \\
\hline C. perfringens ATCC 13124 & 2 & 2 & 2 & 2 & 4 & 4 & 4 & 2 & $1.95^{3}$ \\
\hline C. jejunii ATCC 33291 & 4 & $>4$ & 2 & 4 & 4 & $>4$ & $>4$ & $>4$ & $0.125^{2}$ \\
\hline $\begin{array}{l}\text { S. Typhimurium } \\
\text { ATCC14028 }\end{array}$ & $>4$ & $>4$ & $>4$ & $>4$ & 4 & $>4$ & $>4$ & $>4$ & $0.061^{2}$ \\
\hline E. coli ATCC 25922 & $>4$ & $>4$ & $>4$ & $>4$ & 4 & $>4$ & $>4$ & $>4$ & $0.015^{2}$ \\
\hline P. mirabilis ATCC 12453 & $>4$ & $>4$ & $>4$ & $>4$ & 2 & 2 & $>4$ & $>4$ & $0.03^{2}$ \\
\hline K. pneumoniae ATCC 13883 & $>4$ & $>4$ & $>4$ & $>4$ & 4 & $>4$ & $>4$ & $>4$ & $0.122^{2}$ \\
\hline P. aeruginosa ATCC 90271 & $>4$ & $>4$ & $>4$ & $>4$ & 4 & $>4$ & $>4$ & $>4$ & $0.488^{2}$ \\
\hline B. fragilis ATCC 10240 & 0.5 & 0.25 & 0.25 & 0.25 & 0.5 & 1 & 1 & 1 & $0.98^{3}$ \\
\hline P. intermedia ATCC 25611 & 0.25 & 0.25 & 0.25 & 0.25 & 0.25 & $>4$ & 0.5 & 0.25 & $0.488^{3}$ \\
\hline F. nucleatum ATCC 25586 & 0.125 & 0.125 & 0.0625 & 0.125 & 0.0625 & 0.125 & 0.25 & 0.0625 & $1.95^{3}$ \\
\hline V. parvula ATCC 10790 & 0.25 & 0.25 & 0.125 & 0.25 & 0.25 & 1 & 1 & 2 & $1.95^{3}$ \\
\hline C glabrata ATCC 90030 & 2 & 2 & 2 & 4 & 2 & 4 & 4 & 2 & $0.24^{4}$ \\
\hline C. albicans ATCC 102231 & 1 & 1 & 0.5 & 1 & 1 & 1 & 2 & 1 & $0.48^{4}$ \\
\hline C. parapsilosis ATCC 22019 & 0.5 & 0.5 & 0.25 & 0.5 & 0.5 & 0.5 & 2 & 0.5 & $0.24^{4}$ \\
\hline
\end{tabular}

Minimum inhibitory concentrations (MIC) present representative values of three determinations; the superscript numbers indicate the use of $\left({ }^{1}\right)$ vancomycin, $\left({ }^{2}\right)$ ciprofloxacin, $\left({ }^{3}\right)$ metronidazole and $\left({ }^{4}\right)$ nystatin as the standard drugs towards Gram-positive bacteria, Gram-negative bacteria, anaerobes and yeasts, respectively. 
Previously, P. nigrum exhibited MIC values within a similar range $(0.0625-0.5 \mathrm{mg} / \mathrm{mL})$ against $S$. aureus, S. epidermidis, Staphylococcus xylosus, B. cereus, E. faecalis, Listeria monocytogenes, P. aeruginosa, Salmonella Typhi, S. Typhimurium or E. coli [21,24-26]. Regarding the antibacterial mechanisms of action, black pepper was shown to induce plasmolysis, decrease cell membrane permeability, inhibit the tricarboxylic acid pathway, increase intracellular pyruvic acid and reduce intracellular ATP levels [25]. The antibacterial properties of P. longum against S. aureus, S. pyogenes, K. pneumoniae [27] and P. retrofractum against B. subtilis, S. aureus, E. faecalis, E. coli, K. pneumonia, P. aeruginosa, S. Typhi, Vibrio parahaemolyticus or C. albicans [28] have also been investigated.

However, to the best of our knowledge, the significant antibacterial activity of Piper spices against the anaerobic pathogens tested in this study has not been previously reported. Due to their slow growth rate, poly microbial nature and high resistance to conventional antimicrobial chemotherapy, the management of anaerobic infections is very challenging. Actinomyces spp. are often responsible for head and neck infections, aspiration pneumonia, intracranial abscesses or chronic mastoiditis, whereas Bacteroides spp. are predominant in intra-abdominal and other infections that originate from the gut flora. Prevotella spp. have been isolated from respiratory infections and their complications, while Veillonella spp. have been involved in periodontitis, osteomyelitis or endocarditis [29,30].

\subsection{Antioxidant Activity}

Used for more than 50 years, synthetic antioxidants, such as tert-butylhydroquinone, propylgallate or butylated hydroxytoluene, are nowadays incriminated for carcinogenicity and banned in numerous countries [31,32]. Consequently, there is a high demand, especially from the food industry, for novel natural antioxidants as food preservatives. Previously, different Piper extracts have been shown to possess promising antioxidant effects, as evaluated by DPPH radical, superoxide radical, hydrogen peroxide, nitric oxide scavenging, FRAP, MCA, PBD or $\beta$-carotene bleaching assays [21,33-39].

In the current study, the antioxidant activity of the eight methanolic fruits extracts of Piper spp. was investigated through radical scavenging, metal chelating, metal reducing and PBD assays. Green and red peppers exhibited the most potent DPPH and ABTS radical scavenging activities $(82.44$ and $63.67 \mathrm{mg} \mathrm{TE} / \mathrm{g}$ in DPPH assay for green and red peppers, respectively; 77.60 and $61.63 \mathrm{mg}$ TE/g in ABTS assay for green and red peppers, respectively) (Figure 1a). The scavenging activity of the other samples decreased in the following order: black pepper $1>$ white pepper $>$ black pepper $2>$ black pepper 3 $>$ P. retrofractum $>$ P. longum. A similar trend was observed in metal reducing assays (Figure 1b). CUPRAC varied from $37.36 \mathrm{mg}$ TE/g in black pepper 3 to $140.52 \mathrm{mg} / \mathrm{g}$ TE in green pepper, whereas FRAP varied from $16.05 \mathrm{mg}$ TE/g in P. longum and $77.00 \mathrm{mg} / \mathrm{g}$ TE in green pepper. In MCA (Figure 1c), only black pepper 1 (21.54 mg EDTAE/g), green pepper (4.64 mg EDTAE/g), black pepper 2 (12.35 $\mathrm{mg}$ EDTAE/g) and P. retrofractum (34.80 mg EDTAE/g) were found to be active. PBD assay (Figure 1d) revealed good total antioxidant properties of all investigated spices, with green pepper $(1.35 \mathrm{mmol} \mathrm{TE} / \mathrm{g})$ and black pepper 1 (1.24 mmol TE/g) as the most potent spices.

With respect to the phytochemical composition (Table 1), it can be noticed that the most active samples in DPPH, ABTS, FRAP, CUPRAC and PBD assays were those with the highest TPC, whereas the four active samples in MCA were represented by those with the highest TFC; this could suggest the involvement of these two groups of specialized metabolites in the observed antioxidant properties. 


\section{Radical scavenging}

口DPPH 口ABTS

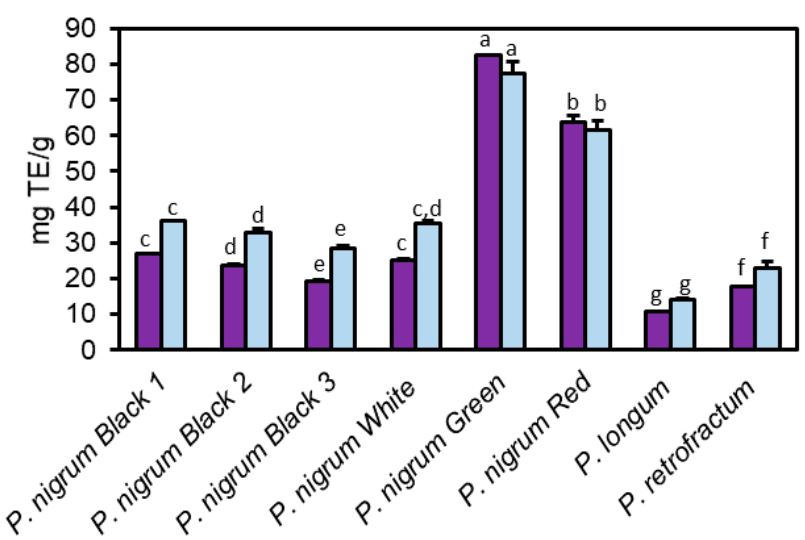

(a)

\section{Metal chelating}

口MCA

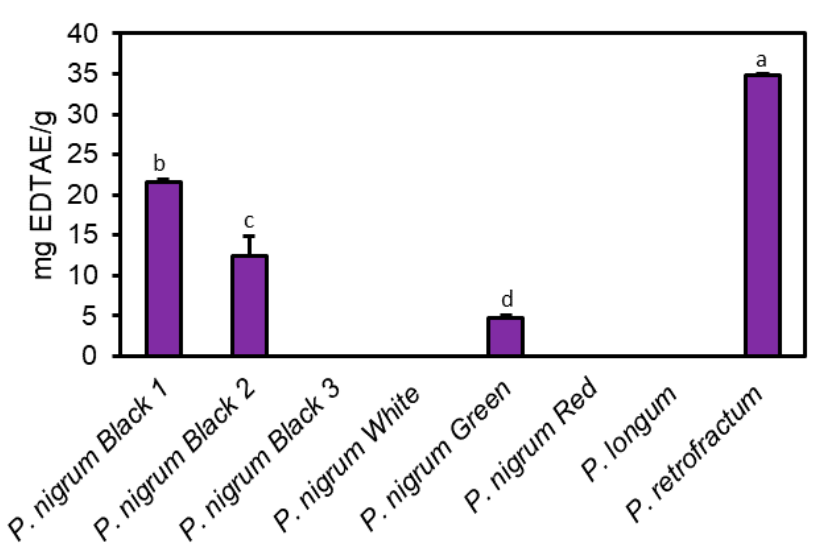

(c)
Metal reducing

口CUPRAC $\square$ FRAP

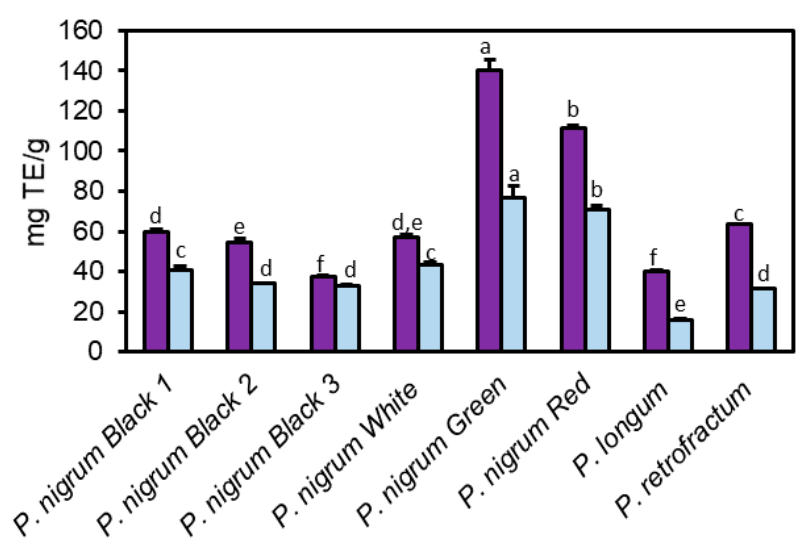

(b)

Total antioxidant

口PBD

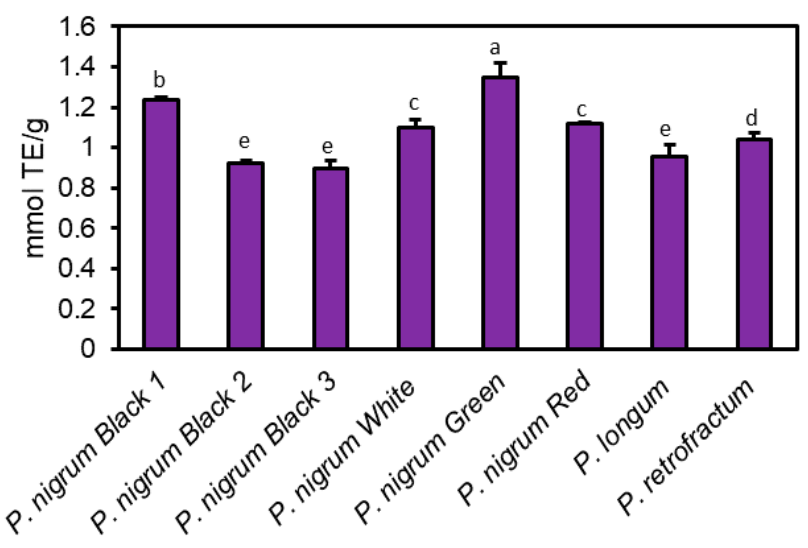

(d)

Figure 1. Antioxidant activity of the methanolic fruit extracts obtained from different spices of genus Piper in (a) radical scavenging, (b) metal reducing, (c) metal chelating and (d) total antioxidant assays. Data are presented as mg Trolox equivalents (TE)/g extract in 1,1-diphenyl-2-picrylhydrazyl (DPPH), 2,2'-azino-bis(3-ethylbenzothiazoline) 6-sulfonic acid (ABTS) radical scavenging, cupric reducing antioxidant capacity (CUPRAC) and ferric reducing antioxidant power (FRAP) assays, mg EDTA equivalents (EDTAE)/g extract in metal chelating assay (MCA) and mmol TE/g extract in phosphomolybdenum (PBD) assay. Each bar represents mean \pm S.D. of three determinations; samples sharing different superscript letters are significantly different at $p<0.05$.

\subsection{Anti-Cholinesterase Activity}

Alzheimer's disease (AD) is the most common cause of dementia and mainly affects the elderly population, provoking progressive cognitive decline, memory impairment and psycho-behavioral disorders. The presence of senile plaques, neurofibrillary tangles as well as a decrease in the cholinergic transmission are often noticed in the brain of patients with $\mathrm{AD}[40,41]$. The inhibition of $\mathrm{AChE}$ and $\mathrm{BChE}$, two sister enzymes that are responsible for the cleavage of the neurotransmitter acetylcholine, is one of the main currently investigated therapeutic strategies, with numerous natural products displaying promising anti-cholinesterase activity [42].

Within the frame of this study, all Piper spices exhibited moderate anti-BChE effects, with values ranging from $0.60 \mathrm{mg}$ GALAE/g (in P. retrofractum) to $3.11 \mathrm{mg}$ GALAE/g (in 
black pepper 3). In contrast, slightly lower anti-AChE properties were noticed, especially considering that, three spices (black peppers 2 and 3 and green pepper) were inactive, with highest activity observed at $2.35 \mathrm{mg}$ GALAE/g in red pepper (Figure 2a). The anticholinesterase properties of the genus Piper have scarcely been reported. For instance, the ethyl acetate extract of P. nigrum exhibited efficient inhibition percentages of $61.5 \%$ against AChE and $90.6 \%$ against $\mathrm{BChE}$ at $100 \mu \mathrm{g} / \mathrm{mL}$, whereas from the 21 isolated piperamides, piperine was the most potent inhibitor with $\mathrm{IC}_{50}$ values of $63.16 \mu \mathrm{g} / \mathrm{mL}$ in $\mathrm{AChE}$ and $25.11 \mu \mathrm{g} / \mathrm{mL}$ in BChE, respectively [43]. On the other hand, the dichloromethane extract of P. longum, as well as piperine $\left(\mathrm{IC}_{50}=0.32 \mathrm{mM}\right)$, pipercide $\left(\mathrm{IC}_{50}=0.61 \mathrm{mM}\right)$ and guineensine $\left(\mathrm{IC}_{50}=1.61 \mathrm{mM}\right)$, were shown to be able to inhibit $\mathrm{AChE}$ [44]. In another study, the methanolic extracts obtained from the fruits of $P$. nigrum and P. retrofractum displayed $\mathrm{IC}_{50}$ values of 11.13 and $14.08 \mu \mathrm{g} / \mathrm{mL}$ against $\mathrm{AChE}$, respectively [45].

AChE and BChE inhibition

口AChE 口BChE

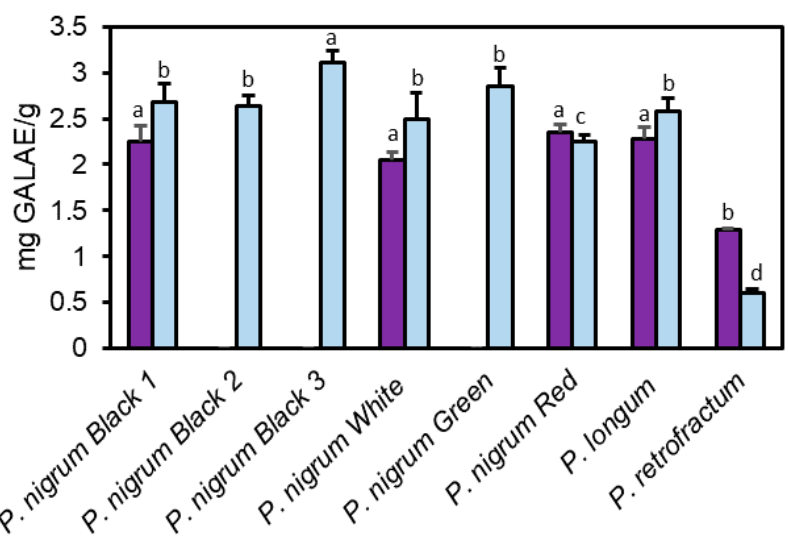

(a)
Amylase and glucosidase inhibition

口Amylase $\square$ Glucosidase

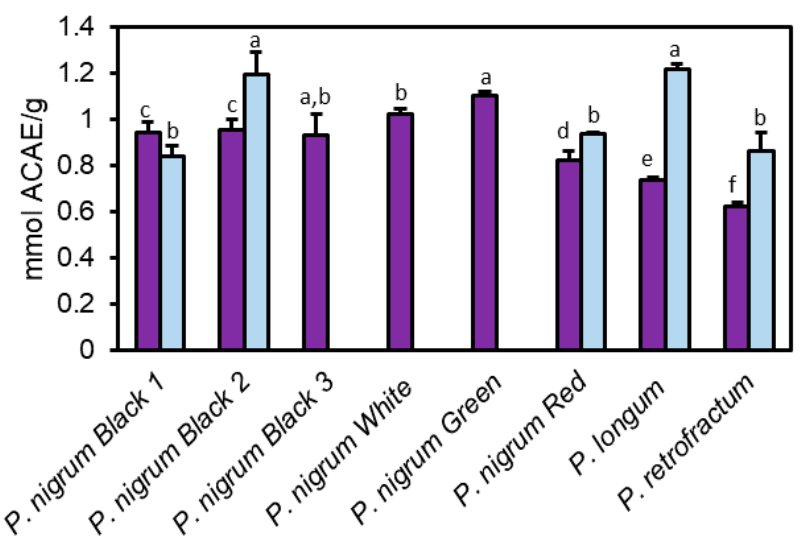

(b)

Figure 2. Acetylcholinesterase, butyrylcholinesterase, glucosidase and amylase inhibitory activity of the methanolic fruit extracts obtained from different spices of genus Piper in (a) cholinesterase and (b) amylase and glucosidase assays. Data are presented as mg galanthamine equivalents (GALAE)/g extract in acetylcholinesterase (AChE) and butyrylcholinesterase (BChE) assays and mg acarbose equivalents (ACAE)/g extract in amylase and glucosidase assays. Each bar represents mean \pm S.D. of three determinations; samples sharing different superscript letters are significantly different at $p<0.05$.

\subsection{Anti-Amylase and Anti-Glucosidase Activity}

Type 2 diabetes mellitus is a chronic metabolic disorder characterized by high blood glucose levels as a consequence of an inefficient insulin functionality or secretion [46]. Inhibiting amylase and glucosidase, two key pancreatic enzymes that convert dietary polysaccharides into absorbable monosaccharides, represents a promising therapeutic approach. Several synthetic inhibitors (acarbose, voglibose, miglitol) are currently available, but they are often reported to produce serious side effects (flatulence, abdominal pain, diarrhea, and hepatotoxicity) [47]. Plants have been shown to be inexhaustible reservoirs of bioactive molecules with potential inhibitory activity against the two enzymes targeted in the management of type 2 diabetes [48]. As presented in Figure 2b, it can be noticed that black pepper 3 , white pepper and green pepper were ineffective against glucosidase, whereas the activity of the remaining spices varied from $0.84 \mathrm{mmol}$ ACAE/g (black pepper 1) to $1.22 \mathrm{mmol} \mathrm{ACAE} / \mathrm{g}$ (P. longum). The extracts displayed anti-amylase effects in the following order: P. retrofractum $<P$. longum $<$ red pepper $<$ black peppers $1-3<$ white pepper < green pepper. Previously, the ethanolic extract of P. nigrum and methanolic extract of $P$. longum were reported to possess anti-glucosidase activities, with $\mathrm{IC}_{50}$ values of 216 and $112.90 \mu \mathrm{g} / \mathrm{mL}$, respectively [47,49]. In addition, various piperamides isolated from $P$. nigrum, $P$. longum or $P$. retrofractum, such as piperine, pipercyclobutanamide $\mathrm{D}$, 
piperoside, pellitorine, brachystamide $B$, guineensine, and pipataline, were shown to exert significant to moderate glucosidase and, sometimes, amylase inhibitory effects $[46,49,50]$.

\subsection{Anti-Melanogenic Activity}

Synthetized by the melanocytes and then transferred to keratinocytes, melanin is the main pigment in hair, skin and eyes. Nevertheless, the excessive production and accumulation of melanin can lead to hyperpigmentation disorders, such as melasma, senile lentigo or freckles [51,52]. Modulation of the melanogenesis by skin-lightening agents represents a promising therapeutic approach. However, the available drugs, such as kojic acid, arbutin, or hydroquinone, are banned in numerous countries, due to their serious side effects, such as irreversible depigmentation, dermatitis or skin cancer [52,53]. Therefore, there is an urgent need to screen for new safer depigmentation agents.

The potential melanin inhibitory effects of spices from the genus Piper were investigated in $\alpha \mathrm{MSH}$-stimulated murine melanoma B16F10 cells. Prior to the melanin assay, the influence of the Piper extracts on the viability of B16F10 cells and immortalized human Ha$\mathrm{CaT}$ keratinocytes was investigated by the neutral red uptake assay. Despite a pronounced cytotoxicity, especially at higher concentrations $(100-200 \mu \mathrm{g} / \mathrm{mL})$, no significant changes in either B16F10 or HaCaT cell viability was noticed at $10 \mu \mathrm{g} / \mathrm{mL}$ (Figure 3); therefore, this concentration was considered safe and selected as test concentration for the melanin assay.

B16F10 cells

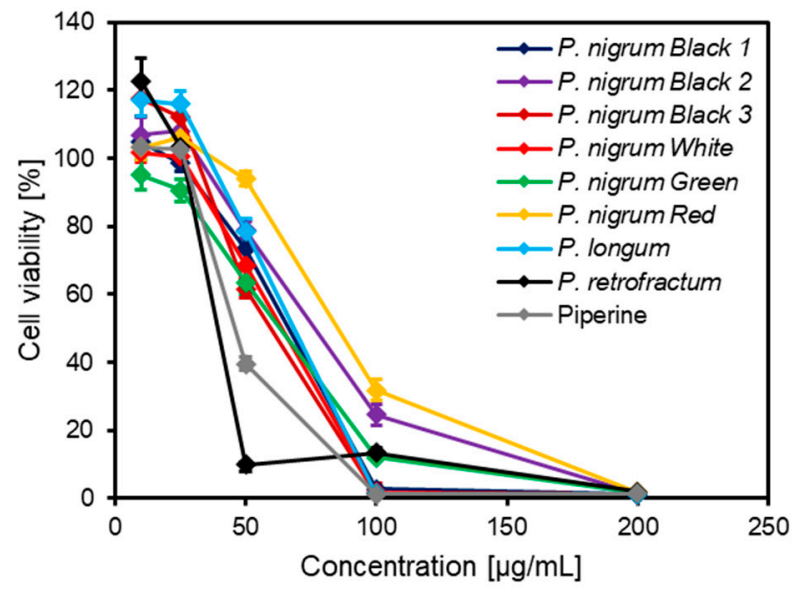

(a)

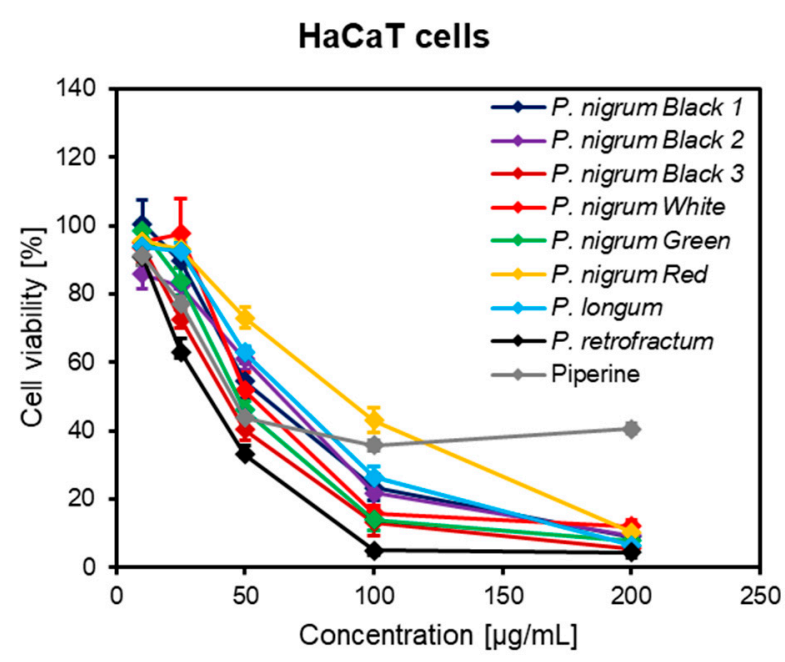

(b)

Figure 3. Concentration-response curves (10-200 $\mu \mathrm{g} / \mathrm{mL})$ showing the effect of the methanolic fruit extracts obtained from different spices of genus Piper and piperine in (a) B16F10 and (b) HaCaT cells. Cells were seeded in 96-well plates $\left(3 \times 10^{3} /\right.$ well $)$ and incubated for $72 \mathrm{~h}$ (B16F10 cells) or $48 \mathrm{~h}$ (HaCaT cells) with different concentrations of Piper extracts $(10-200 \mu \mathrm{g} / \mathrm{mL})$ or piperine $(10-200 \mu \mathrm{g} / \mathrm{mL})$. Data are presented as percentage cell viability $(\%)$ in comparison to the solvent (DMSO)-treated control cells, set at $100 \%$.

As compared to the non-treated cells ( $\alpha \mathrm{MSH}-$ ), the extracellular melanin content (=melanin release in the conditioned medium) and intracellular melanin content (=melanin content in the lysate) were both significantly increased by 2.7- and 4.3-fold in $\alpha \mathrm{MSH}+$ cells, respectively (Figure 4$)$. When $\alpha \mathrm{MSH}+$ cells were treated with Piper samples $(10 \mu \mathrm{g} / \mathrm{mL})$, a significant reduction of intracellular melanin content was noticed in white pepper $(47.72 \%$ of $\alpha \mathrm{MSH}+$ cells), green pepper (60.65\% of $\alpha \mathrm{MSH}+$ cells) and, especially, P. longum (32.05\% $\alpha \mathrm{MSH}+$ cells). Furthermore, the same extracts also decreased the melanin release, with a relative extracellular melanin content of $38.06 \%, 40.68 \%$ and $45.78 \%$ of $\alpha \mathrm{MSH}+$ cells, respectively. Piperine $(10 \mu \mathrm{g} / \mathrm{mL})$ was only able to reduce the intracellular melanin content (61.01\% of $\alpha \mathrm{MSH}+$ cells), whereas the extracellular melanin release was not considerably impaired. Kojic acid $(10 \mu \mathrm{g} / \mathrm{mL})$, the positive control for the melanin assay, significantly 
downregulated both the intracellular (61.13\% of $\alpha \mathrm{MSH}+$ cells) and extracellular (68.59\% of $\alpha \mathrm{MSH}+$ cells) melanin content.

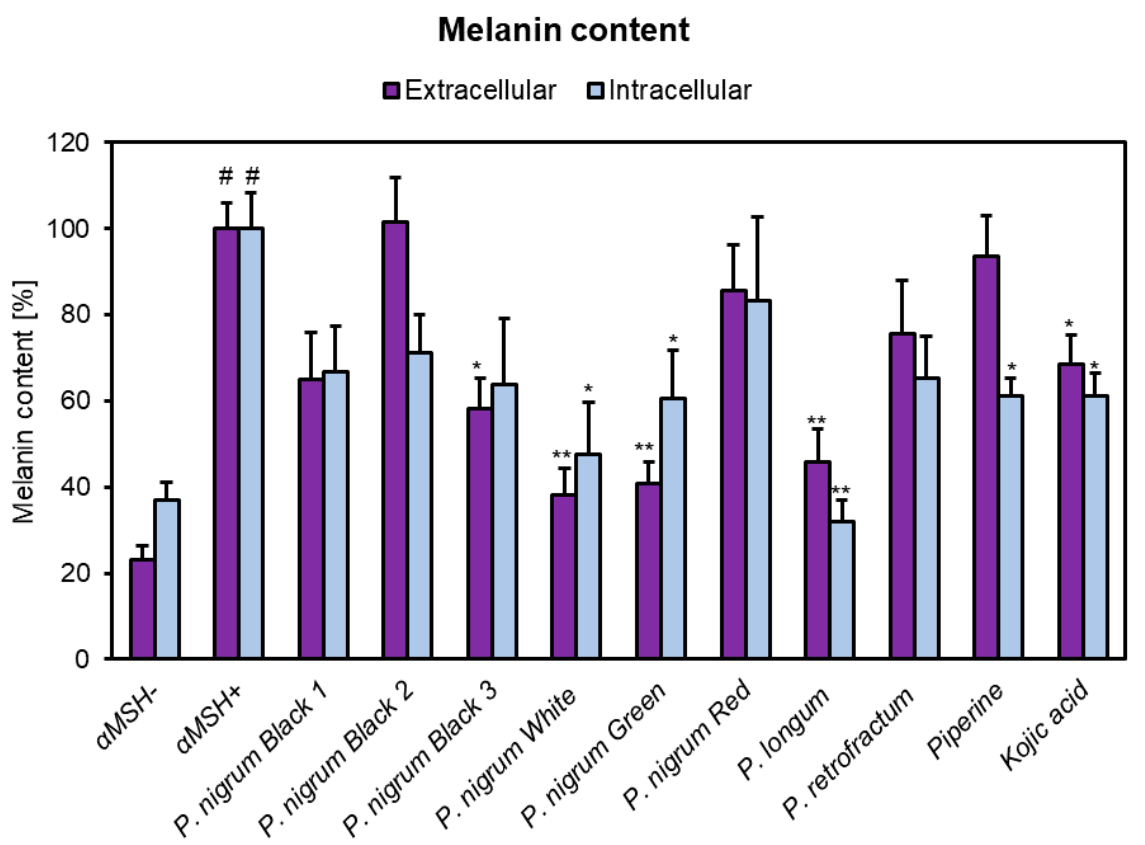

Figure 4. Melanin inhibitory activity in $\alpha \mathrm{MSH}$-stimulated B16F10 cells of the methanolic fruit extracts obtained from different spices of genus Piper, piperine and kojic acid. B16F10 cells were seeded in 6-well plates $\left(0.5 \times 10^{5} /\right.$ well $)$ and incubated for $72 \mathrm{~h}$ with Piper extracts $(10 \mu \mathrm{g} / \mathrm{mL})$, piperine $(10 \mu \mathrm{g} / \mathrm{mL})$ or kojic acid $(10 \mu \mathrm{g} / \mathrm{mL})$; melanin production was stimulated with $\alpha \mathrm{MSH}(10 \mathrm{nM})$. Data are presented as the percentage of intracellular and extracellular melanin content in comparison to $\alpha \mathrm{MSH}$ stimulated control $(\alpha \mathrm{MSH}+)$ cells, set at $100 \%$. Each bar represents the mean \pm S.E.M. of at least three independent experiments performed in triplicates; \# $p<0.001$ vs. non-stimulated ( $\alpha \mathrm{MSH}-)$ cells; ${ }^{*} p<0.05 ;{ }^{* *} p<0.01 ;$ vs. $\alpha \mathrm{MSH}+$ cells.

To the best of our knowledge, there are no previous studies reporting on the antimelanogenic activity of extracts obtained from various Piper spices. Nevertheless, the potential of different piperamides to inhibit the melanin production in $\alpha \mathrm{MSH}$-stimulated B16F10 cells has been documented. For instance, piperlongumine significantly reduced the total melanin production, by reducing the activity of tyrosinase and expression of several pro-melanogenic proteins, such as tyrosinase-related protein-1 (TRP-1), TRP2, microphthalmia-associated transcription factor (MITF) [54]. On the other hand, piperlonguminine decreased the production of melanin by downregulating tyrosine expression at the transcription level via a reduction of cyclic adenosine monophosphate responsive element binding protein (CREB) phosphorylation $[55,56]$.

\subsection{Anti-Tyrosinase Activity}

To investigate whether the anti-melanogenic effects of Piper spices in $\alpha \mathrm{MSH}$-stimulated B16F10 cells might be exerted via tyrosinase inhibition, monophenolase and diphenolase assays were performed. Tyrosinase is a key enzyme of melanogenesis, catalyzing the rate-limiting conversion of L-tyrosine to L-dihydroxyphenylalanine (L-DOPA) and subsequently to dopaquinone. Numerous plant extracts have previously been shown to exert tyrosinase inhibitory effects [20,57-59]. The influence on the monophenolase activity was investigated with mushroom tyrosinase (by adding L-tyrosine as substrate), whereas the influence on the diphenolase activity was evaluated with both mushroom and murine tyrosinase (by adding L-DOPA as substrate) (Figure 5). It was noticed that only the three black pepper samples $(10 \mu \mathrm{g} / \mathrm{mL})$ significantly reduced the mushroom monophenolase activity (by 22-27\%). With respect to the mushroom diphenolase, black, green and white 
peppers showed moderate anti-tyrosinase effects (reductions by 13-16\%). On the other hand, the activity of murine tyrosinase was significantly impaired by black (1-2) and white peppers and, additionally, by P. longum (activities between 78.36-85.22\%). Piperine was unable to modulate the tyrosinase activity, whereas kojic acid, the positive control, decreased the mushroom monophenolase and diphenolase activity to $30.86 \%$ and $57.27 \%$, respectively, with no significant effects on murine tyrosinase. Previously, the anti-tyrosinase activity of various Piper species, such as P. retrofractum, P. caninum Blume, P. abbreviatum Opiz, P. erecticaule C.DC., P. stylosum Miq., P. magnibaccum C.DC., and P. betle L., has been reported [60-63].

\section{Tyrosinase inhibition}

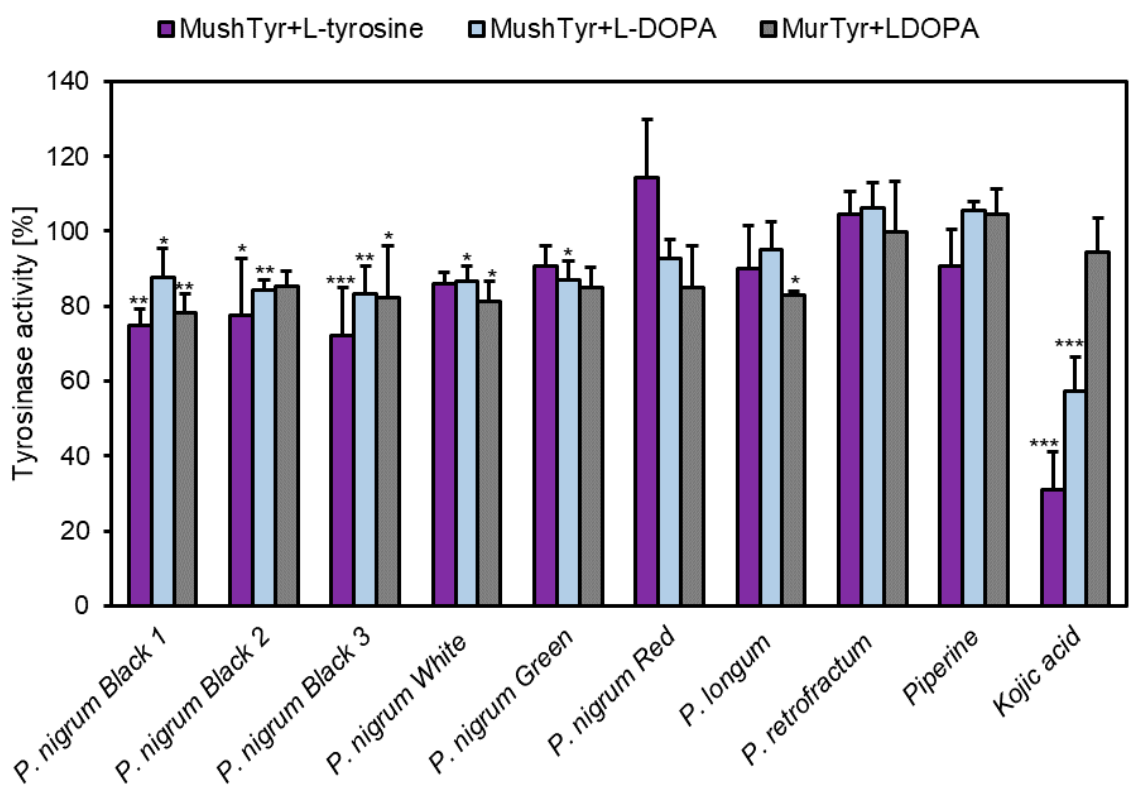

Figure 5. Tyrosinase inhibitory activity of the methanolic fruit extracts $(10 \mu \mathrm{g} / \mathrm{mL})$ obtained from different spices of genus Piper, piperine $(10 \mu \mathrm{g} / \mathrm{mL})$ and kojic acid $(10 \mu \mathrm{g} / \mathrm{mL})$. Data are presented as percentage of tyrosinase activity in comparison to solvent (DMSO) control, set at $100 \%$. Each bar represents the mean \pm S.D. of three determinations; ${ }^{*} p<0.05 ;{ }^{* *} p<0.01$; ${ }^{* *} p<0.001$ vs. control.

It is worth mentioning that the decrease in the melanin content in B16F10 cells treated with Piper extracts might result not only from the direct modulation of tyrosinase activity, but also from the transcriptional control of genes involved in the melanogenesis pathway or post-transcriptional modification of tyrosinase leading to enzyme degradation [64]. The potential involvement of Piper compounds in these pathways requires further investigation.

\section{Conclusions}

In this work, the phytochemical and multifunctional biological profiling of four varieties of $P$. nigrum (black, green, white and red) and two other Piper species, namely P. longum and P. retrofractum, was presented. Firstly, a comprehensive metabolite characterization by TPC, TFC, LC-HRMS/MS and LC-DAD was performed, revealing significant levels of bioactive piperamides, such as piperine, piperlongumine, piperlonguminine, retrofractamide $\mathrm{B}$, guineensine, pipernonaline, or $N$-isobutyl-2,4,12-octadecatrienamide. Then, the spices were assessed for their antibacterial and antifungal properties on a panel of 22 reference microorganisms. Beside a moderate activity against Staphylococcus aureus, S. epidermidis, M. luteus, B. cereus and C. parapsilosis (MIC $=0.125-0.5 \mathrm{mg} / \mathrm{mL}$ ), several samples, such as black, green, white peppers or $P$. retrofractum, were particularly active $(\mathrm{MIC}=0.0625-0.125 \mathrm{mg} / \mathrm{mL})$ against anaerobe species, such as $A$. israelii, $B$. fragilis, $P$. intermedia, F. nucleatum and V. parvula. A potent antioxidant activity of the investigated spices was revealed, as evaluated by several complementary tests, such as radical scavenging, 
metal reducing, chelating and phosphomolybdenum assays. With respect to the modulation of enzymes targeted in the management of Alzheimer's disease (acetylcholinesterase, butyrylcholinesterase) and type 2 diabetes mellitus (amylase, glucosidase), the current data suggest moderate inhibitory effects of Piper spices against the investigated enzymes. Several Piper extracts, namely white pepper, green pepper and P. longum, inhibited both the melanin synthesis (to $32.05-60.65 \%$ of $\alpha$-MSH+ cells) and melanin release $(38.06-45.78 \%$ of $\alpha$-MSH+ cells) in $\alpha$-MSH-stimulated B16F10 cells at $10 \mu \mathrm{g} / \mathrm{mL}$, a non-toxic concentration in both B16F10 and HaCaT cells. The anti-melanogenic properties could be partly explained by the moderate inhibition of murine tyrosinase (tyrosinase activities between 78.36-85.22\%).

By evaluating the poly pharmacological potential of a series of Piper varieties and species, globally available and used as spices, our study shows similarities and differences between the investigated Piper samples. This could inspire future research into the use of Piper nutraceuticals or cosmeceuticals in the management of infectious diseases, Alzheimer's dementia, type 2 diabetes mellitus or hyperpigmentation disorders.

Author Contributions: Conceptualization, S.V.L., A.T. and K.S.-W.; methodology, S.V.L., K.G.-B., G.Z. and I.K.-G.; software, S.V.L., K.G.-B., G.Z., K.C. and I.K.-G.; validation, S.V.L., K.G.-B., A.T., G.Z., I.K.-G. and K.S.-W.; formal analysis, S.V.L., K.G.-B., G.Z., K.C. and I.K.-G.; investigation, S.V.L., K.G.-B., M.S.-G., K.C. and I.K.-G.; resources, S.V.L., K.G.-B., G.Z., I.K.-G., M.M. and K.S.-W.; data curation, S.V.L., G.Z., J.G.; writing—original draft preparation, S.V.L. and A.T.; writing-review and editing, K.G.-B., G.Z., I.K.-G., M.M., J.G., K.S.-W.; visualization, S.V.L.; supervision, K.G.-B., G.Z., M.M. and K.S.-W.; project administration, S.V.L.; funding acquisition, K.G.-B., I.K.-G., K.S.-W. All authors have read and agreed to the published version of the manuscript.

Funding: This research was funded by the statutory funds of the Medical University of Lublin, Poland (DS 28) and statutory project of the University of Information Technology and Management in Rzeszow, Poland (DS 503-07-01-38).

Institutional Review Board Statement: Not applicable.

Informed Consent Statement: Not applicable.

Data Availability Statement: Data is contained within the article.

Conflicts of Interest: The authors declare no conflict of interest.

\section{References}

1. Mgbeahuruike, E.E.; Yrjönen, T.; Vuorela, H.; Holm, Y. Bioactive compounds from medicinal plants: Focus on Piper species. S. Afr. J. Bot. 2017, 112, 54-69. [CrossRef]

2. Luca, S.V.; Minceva, M.; Gertsch, J.; Skalicka-Woźniak, K. LC-HRMS/MS-based phytochemical profiling of Piper spices: Global association of piperamides with endocannabinoid system modulation. Food Res. Int. 2021, 141, 110123. [CrossRef]

3. Takooree, H.; Aumeeruddy, M.Z.; Rengasamy, K.R.; Venugopala, K.N.; Jeewon, R.; Zengin, G.; Mahomoodally, M.F. A systematic review on black pepper (Piper nigrum L.): From folk uses to pharmacological applications. Crit. Rev. Food Sci. Nutr. 2019, 59, S210-S243. [CrossRef] [PubMed]

4. Salehi, B.; Zakaria, Z.A.; Gyawali, R.; Ibrahim, S.A.; Rajkovic, J.; Shinwari, Z.K.; Khan, T.; Sharifi-Rad, J.; Ozleyen, A.; Turkdonmez, E. Piper species: A comprehensive review on their phytochemistry, biological activities and applications. Molecules 2019, $24,1364$. [CrossRef] [PubMed]

5. Kumar, S.; Kamboj, J.; Sharma, S. Overview for various aspects of the health benefits of Piper longum Linn. fruit. J. Acupunct. Meridian Stud. 2011, 4, 134-140. [CrossRef]

6. Yadav, V.; Krishnan, A.; Vohora, D. A systematic review on Piper longum L.: Bridging traditional knowledge and pharmacological evidence for future translational research. J. Ethnopharmacol. 2020, 247, 112255. [CrossRef] [PubMed]

7. Islam, M.T.; Hasan, J.; Snigdha, H.S.H.; Ali, E.S.; Sharifi-Rad, J.; Martorell, M.; Mubarak, M.S. Chemical profile, traditional uses, and biological activities of Piper chaba Hunter: A review. J. Ethnopharmacol. 2020, 257, 112853. [CrossRef] [PubMed]

8. Shityakov, S.; Bigdelian, E.; Hussein, A.A.; Hussain, M.B.; Tripathi, Y.C.; Khan, M.U.; Shariati, M.A. Phytochemical and pharmacological attributes of piperine: A bioactive ingredient of black pepper. Eur. J. Med. Chem. 2019, 176, 149-161. [CrossRef]

9. Kim, N.; Nam, M.; Kang, M.S.; Lee, J.O.; Lee, Y.W.; Hwang, G.-S.; Kim, H.S. Piperine regulates UCP1 through the AMPK pathway by generating intracellular lactate production in muscle cells. Sci. Rep. 2017, 7, 1-13. [CrossRef]

10. Matsuda, D.; Ohte, S.; Ohshiro, T.; Jiang, W.; Rudel, L.; Hong, B.; Si, S.; Tomoda, H. Molecular target of piperine in the inhibition of lipid droplet accumulation in macrophages. Biol. Pharm. Bull. 2008, 31, 1063-1066. [CrossRef] 
11. Lee, S.W.; Rho, M.-C.; Park, H.R.; Choi, J.-H.; Kang, J.Y.; Lee, J.W.; Kim, K.; Lee, H.S.; Kim, Y.K. Inhibition of diacylglycerol acyltransferase by alkamides isolated from the fruits of Piper longum and Piper nigrum. J. Agric. Food Chem. 2006, 54, 9759-9763. [CrossRef] [PubMed]

12. Reynoso-Moreno, I.s.; Najar-Guerrero, I.; Escarenño, N.; Flores-Soto, M.E.; Gertsch, J.r.; Viveros-Paredes, J.M. An endocannabinoid uptake inhibitor from black pepper exerts pronounced anti-inflammatory effects in mice. J. Agric. Food Chem. 2017, 65, 9435-9442. [CrossRef]

13. Nicolussi, S.; Viveros-Paredes, J.M.; Gachet, M.S.; Rau, M.; Flores-Soto, M.E.; Blunder, M.; Gertsch, J. Guineensine is a novel inhibitor of endocannabinoid uptake showing cannabimimetic behavioral effects in BALB/c mice. Pharmacol. Res. 2014, 80, 52-65. [CrossRef] [PubMed]

14. Uysal, S.; Zengin, G.; Locatelli, M.; Bahadori, M.B.; Mocan, A.; Bellagamba, G.; De Luca, E.; Mollica, A.; Aktumsek, A. Cytotoxic and enzyme inhibitory potential of two Potentilla species (P. speciosa L. and P. reptans Willd.) and their chemical composition. Front. Pharmacol. 2017, 8, 290. [CrossRef] [PubMed]

15. Grochowski, D.M.; Uysal, S.; Aktumsek, A.; Granica, S.; Zengin, G.; Ceylan, R.; Locatelli, M.; Tomczyk, M. In vitro enzyme inhibitory properties, antioxidant activities, and phytochemical profile of Potentilla thuringiaca. Phytochem. Lett. 2017, 20, 365-372. [CrossRef]

16. European Committee for Antimicrobial Susceptibility Testing (EUCAST) of the European Society of Clinical Microbiology and Infectious Diseases (ESCMID). Determination of minimum inhibitory concentrations (MICs) of antibacterial agents by broth dilution. Clin. Microbiol. Infect. 2003, 9, 9-15.

17. Repetto, G.; Del Peso, A.; Zurita, J.L. Neutral red uptake assay for the estimation of cell viability/cytotoxicity. Nat. Protoc. 2008, 3, 1125-1131. [CrossRef]

18. Bradford, M.M. A rapid and sensitive method for the quantitation of microgram quantities of protein utilizing the principle of protein-dye binding. Anal. Biochem. 1976, 72, 248-254. [CrossRef]

19. Uchida, R.; Ishikawa, S.; Tomoda, H. Inhibition of tyrosinase activity and melanine pigmentation by 2-hydroxytyrosol. Acta Pharm. Sin. B 2014, 4, 141-145. [CrossRef]

20. Sabitov, A.; Gaweł-Bęben, K.; Sakipova, Z.; Strzępek-Gomółka, M.; Hoian, U.; Satbayeva, E.; Głowniak, K.; Ludwiczuk, A. Rosa platyacantha Schrenk from Kazakhstan-Natural source of bioactive compounds with cosmetic significance. Molecules 2021, 26, 2578. [CrossRef]

21. Zarai, Z.; Boujelbene, E.; Salem, N.B.; Gargouri, Y.; Sayari, A. Antioxidant and antimicrobial activities of various solvent extracts, piperine and piperic acid from Piper nigrum. Lwt-Food Sci. Technol. 2013, 50, 634-641. [CrossRef]

22. Trifan, A.; Luca, S.V.; Greige-Gerges, H.; Miron, A.; Gille, E.; Aprotosoaie, A.C. Recent advances in tackling microbial multidrug resistance with essential oils: Combinatorial and nano-based strategies. Crit. Rev. Microbiol. 2020, 46, 338-357. [CrossRef] [PubMed]

23. Efferth, T.; Kuete, V. Cameroonian medicinal plants: Pharmacology and derived natural products. Front. Pharmacol. 2010, 1, 123.

24. Karsha, P.V.; Lakshmi, O.B. Antibacterial activity of black pepper (Piper nigrum Linn.) with special reference to its mode of action on bacteria. Indian J. Nat. Prod. Res. 2010, 1, 213-215.

25. Zou, L.; Hu, Y.-Y.; Chen, W.-X. Antibacterial mechanism and activities of black pepper chloroform extract. J. Food Sci. Technol. 2015, 52, 8196-8203. [CrossRef] [PubMed]

26. Tang, H.; Chen, W.; Dou, Z.-M.; Chen, R.; Hu, Y.; Chen, W.; Chen, H. Antimicrobial effect of black pepper petroleum ether extract for the morphology of Listeria monocytogenes and Salmonella typhimurium. J. Food Sci. Technol 2017, 54, 2067-2076. [CrossRef]

27. Saraf, A.; Saraf, A. Phytochemical and antimicrobial studies of medicinal plant Piper longum Linn. Int. J. Pharmacogn. Phytochem. Res. 2014, 6, 213-222.

28. Panphut, W.; Budsabun, T.; Sangsuriya, P. In vitro antimicrobial activity of piper retrofractum fruit extracts against microbial pathogens causing infections in human and animals. Int. J. Microbiol. 2020, 2020, 5638961. [CrossRef]

29. Brook, I. Spectrum and treatment of anaerobic infections. J. Infect. Chemother. 2016, 22, 1-13. [CrossRef]

30. Mitsui, T.; Saito, M.; Harasawa, R. Salivary nitrate-nitrite conversion capacity after nitrate ingestion and incidence of Veillonella spp. in elderly individuals. J. Oral Sci. 2018, 60, 405-410. [CrossRef]

31. Aprotosoaie, A.C.; Miron, A.; Ciocârlan, N.; Brebu, M.; Roşu, C.M.; Trifan, A.; Vochiţa, G.; Gherghel, D.; Luca, S.V.; Niţă, A. Essential oils of Moldavian Thymus species: Chemical composition, antioxidant, anti-Aspergillus and antigenotoxic activities. Flav. Fragr. J. 2019, 34, 175-186. [CrossRef]

32. Trifan, A.; Zengin, G.; Sinan, K.I.; Wolfram, E.; Skalicka-Woźniak, K.; Luca, S.V. LC-HRMS/MS phytochemical profiling of Symphytum officinale L. and Anchusa ochroleuca M. Bieb.(Boraginaceae): Unveiling their multi-biological potential via an integrated approach. J. Pharm. Biomed. Anal. 2021, 204, 114283. [CrossRef]

33. Gülçin, İ. The antioxidant and radical scavenging activities of black pepper (Piper nigrum) seeds. Int. J. Food Sci. Nutr. 2005, 56, 491-499. [CrossRef]

34. Agbor, G.A.; Vinson, J.A.; Oben, J.E.; Ngogang, J.Y. In vitro antioxidant activity of three Piper species. J. Herb. Pharmacother. 2008, 7, 49-64. [CrossRef]

35. Chatterjee, S.; Niaz, Z.; Gautam, S.; Adhikari, S.; Variyar, P.S.; Sharma, A. Antioxidant activity of some phenolic constituents from green pepper (Piper nigrum L.) and fresh nutmeg mace (Myristica fragrans). Food Chem. 2007, 101, 515-523. [CrossRef] 
36. Samudram, P.; Vasuki, R.; Rajeshwari, H.; Geetha, A.; Moorthi, P.S. Antioxidant and antihepatotoxic activities of ethanolic crude extract of Melia azedarach and Piper longum. J. Med. Plants Res. 2009, 3, 1078-1083.

37. Barua, C.; Singh, A.; Sen, S.; Barua, A.; Barua, I. In vitro antioxidant and antimycobacterial activity of seeds of Piper longum Linn: A comparative study. SAJ Pharm. Pharmacol. 2014, 1. [CrossRef]

38. Jadid, N.; Hidayati, D.; Hartanti, S.R.; Arraniry, B.A.; Rachman, R.Y.; Wikanta, W. Antioxidant activities of different solvent extracts of Piper retrofractum Vahl. using DPPH assay. Proc. AIP Conf. 2017, 1854, 020019.

39. Mahaldar, K.; Hossain, A.; Islam, F.; Islam, S.; Islam, M.A.; Shahriar, M.; Rahman, M.M. Antioxidant and hepatoprotective activity of Piper retrofractum against paracetamol-induced hepatotoxicity in Sprague-Dawley rat. Nat. Prod. Res. 2020, 34, $3219-3225$. [CrossRef]

40. Dung, H.V.; Cuong, T.D.; Chinh, N.M.; Quyen, D.; Kim, J.A.; Byeon, J.S.; Woo, M.H.; Choi, J.S.; Min, B.S. Compounds from the aerial parts of Piper bavinum and their anti-cholinesterase activity. Arch. Pharm. Res. 2015, 38, 677-682. [CrossRef]

41. Ferreres, F.; Oliveira, A.P.; Gil-Izquierdo, A.; Valentão, P.; Andrade, P.B. Piper betle leaves: Profiling phenolic compounds by HPLC/DAD-ESI/MSn and anti-cholinesterase activity. Phytochem. Anal. 2014, 25, 453-460. [CrossRef] [PubMed]

42. Gök, H.N.; Luca, S.V.; Ay, S.T.; Komsta, Ł.; Salmas, R.E.; Orhan, I.E.; Skalicka-Woźniak, K. Profiling the annual change of the neurobiological and antioxidant effects of five Origanum species in correlation with their phytochemical composition. Food Chem. 2021, 368, 130775. [CrossRef] [PubMed]

43. Tu, Y.; Zhong, Y.; Du, H.; Luo, W.; Wen, Y.; Li, Q.; Zhu, C.; Li, Y. Anticholinesterases and antioxidant alkamides from Piper nigrum fruits. Nat. Prod. Res. 2016, 30, 1945-1949. [CrossRef] [PubMed]

44. Khatami, Z.; Herdlinger, S.; Sarkhail, P.; Zehl, M.; Kaehlig, H.; Schuster, D.; Adhami, H.-R. Isolation and characterization of acetylcholinesterase inhibitors from Piper longum and binding mode predictions. Planta Med. 2020, 86, 1118-1124. [CrossRef]

45. Tappayuthpijarn, P.; Sattaponpan, C.; Sakpakdeecharoen, I.; Ittharat, A. Cholinesterase inhibitory and antioxidant activities of Thai traditional remedies potentially used for Alzheimer's disease. Thai J. East Asian Stud. 2012, 17, 18-25. [CrossRef]

46. Luyen, B.T.T.; Tai, B.H.; Thao, N.P.; Yang, S.Y.; Cuong, N.M.; Kwon, Y.I.; Jang, H.D.; Kim, Y.H. A new phenylpropanoid and an alkylglycoside from Piper retrofractum leaves with their antioxidant and $\alpha$-glucosidase inhibitory activity. Bioorg. Med. Chem. Lett. 2014, 24, 4120-4124. [CrossRef] [PubMed]

47. Magaña-Barajas, E.; Buitimea-Cantúa, G.V.; Hernández-Morales, A.; Torres-Pelayo, V.d.R.; Vázquez-Martínez, J.; Buitimea-Cantúa, N.E. In vitro $\alpha$-amylase and $\alpha$-glucosidase enzyme inhibition and antioxidant activity by capsaicin and piperine from Capsicum chinense and Piper nigrum fruits. J. Environ. Sci. Health B 2021, 56, 282-291. [CrossRef]

48. Gevrenova, R.; Zengin, G.; Sinan, K.I.; Yıldıztugay, E.; Zheleva-Dimitrova, D.; Picot-Allain, C.; Mahomoodally, M.F.; Imran, M.; Dall'Acqua, S. UHPLC-MS Characterization and biological insights of different solvent extracts of two Achillea species (A. aleppica and A. santolinoides) from Turkey. Antioxidants 2021, 10, 1180. [CrossRef]

49. Pullela, S.V.; Tiwari, A.K.; Vanka, U.S.; Vummenthula, A.; Tatipaka, H.B.; Dasari, K.R.; Khan, I.A.; Janaswamy, M.R. HPLC assisted chemobiological standardization of $\alpha$-glucosidase-I enzyme inhibitory constituents from Piper longum Linn-An Indian medicinal plant. J. Ethnopharmacol. 2006, 108, 445-449. [CrossRef]

50. Huu, D.M.N.; Dang, P.H.; Huynh, N.V.; Dang, H.P.; Vuong, L.; Nguyen, T.L.T. Pipercyclobutanamide D, a new member of the cyclobutanamide-type alkaloid, from the roots of Piper nigrum. J. Asian Nat. Prod. Res. 2020, 1-7. [CrossRef]

51. Srisayam, M.; Weerapreeyakul, N.; Kanokmedhakul, K. Inhibition of two stages of melanin synthesis by sesamol, sesamin and sesamolin. Asian Pac. J. Trop. Biomed. 2017, 7, 886-895. [CrossRef]

52. Ullah, S.; Park, C.; Ikram, M.; Kang, D.; Lee, S.; Yang, J.; Park, Y.; Yoon, S.; Chun, P.; Moon, H.R. Tyrosinase inhibition and anti-melanin generation effect of cinnamamide analogues. Bioorg. Chem. 2019, 87, 43-55. [CrossRef] [PubMed]

53. Mustapha, N.; Bzéouich, I.M.; Ghedira, K.; Hennebelle, T.; Chekir-Ghedira, L. Compounds isolated from the aerial part of Crataegus azarolus inhibit growth of B16F10 melanoma cells and exert a potent inhibition of the melanin synthesis. Biomed. Pharmacother. 2015, 69, 139-144. [CrossRef] [PubMed]

54. Jeon, H.-J.; Kim, K.; Kim, Y.-D.; Lee, S.-E. Antimelanogenic activities of piperlongumine derived from Piper longum on murine B16F10 melanoma cells in vitro and zebrafish embryos in vivo: Its molecular mode of depigmenting action. App. Biol. Chem. 2019, 62, 1-7. [CrossRef]

55. Min, K.R.; Kim, K.-S.; Ro, J.S.; Lee, S.H.; Kim, J.A.; Son, J.K.; Kim, Y. Piperlonguminine from Piper longum with inhibitory effects on alpha-melanocyte-stimulating hormone-induced melanogenesis in melanoma B16 cells. Planta Med. 2004, 70, 1115-1118. [CrossRef]

56. Kim, K.S.; Kim, J.A.; Eom, S.Y.; Lee, S.H.; Min, K.R.; Kim, Y. Inhibitory effect of piperlonguminine on melanin production in melanoma B16 cell line by downregulation of tyrosinase expression. Pigment Cell Res. 2006, 19, 90-98. [CrossRef]

57. Strzępek-Gomółka, M.; Gaweł-Bęben, K.; Angelis, A.; Antosiewicz, B.; Sakipova, Z.; Kozhanova, K.; Głowniak, K.; Kukula-Koch, W. Identification of mushroom and murine tyrosinase inhibitors from Achillea biebersteinii Afan. extract. Molecules 2021, 26, 964. [CrossRef]

58. Gaweł-Bęben, K.; Strzępek-Gomółka, M.; Czop, M.; Sakipova, Z.; Głowniak, K.; Kukula-Koch, W. Achillea millefolium L. and Achillea biebersteinii Afan. hydroglycolic extracts-bioactive ingredients for cosmetic use. Molecules 2020, 25, 3368. [CrossRef]

59. Gaweł-Bęben, K.; Kukula-Koch, W.; Hoian, U.; Czop, M.; Strzępek-Gomółka, M.; Antosiewicz, B. Characterization of Cistus $\times$ incanus L. and Cistus ladanifer L. extracts as potential multifunctional antioxidant ingredients for skin protecting cosmetics. Antioxidants 2020, 9, 202. [CrossRef] 
60. Salleh, W.M.N.H.W.; Hashim, N.A.; Ahmad, F.; Yen, K.H. Anticholinesterase and antityrosinase activities of ten Piper species from Malaysia. Adv. Pharm. Bull. 2014, 4, 527.

61. Salleh, W.; Ahmad, F.; Khong, H. Antioxidant and anti-tyrosinase activities from Piper officinarum C. DC (Piperaceae). J. Appl. Pharm. Sci. 2014, 4, 087-091.

62. Hashim, N.A.; Ahmad, F.; Salleh, W.M.N.H.W.; Khamis, S. Phytochemicals and tyrosinase inhibitory activity from Piper caninum and Piper magnibaccum. Pharm. Sci. 2019, 25, 358-363. [CrossRef]

63. Tan, Y.P.; Chan, E.W.C. Antioxidant, antityrosinase and antibacterial properties of fresh and processed leaves of Anacardium occidentale and Piper betle. Food Biosci. 2014, 6, 17-23. [CrossRef]

64. Qian, W.; Liu, W.; Zhu, D.; Cao, Y.; Tang, A.; Gong, G.; Su, H. Natural skin-whitening compounds for the treatment of melanogenesis (Review). Exp. Ther. Med. 2020, 20, 173-185. [CrossRef] 